\title{
Monitoring the Athlete Match Response: Can External Load Variables Predict Post- match Acute and Residual Fatigue in Soccer? A Systematic Review with Meta- analysis
}

Karim Hader ${ }^{1,2}$, Michael C. Rumpf ${ }^{3,4}$, Maxime Hertzog ${ }^{1}$, Liam P. Kilduff ${ }^{5,6}$, Olivier Girard ${ }^{7}$ and Joao R. Silva ${ }^{1,8^{*}}$ (D)

\begin{abstract}
Background: Monitoring athletes' external load during a soccer match may be useful to predict post-match acute and residual fatigue. This estimation would allow individual adjustments to training programs to minimize injury risk, improve well-being, and restore players' physical performance and inform the recovery process.

Methods: Using a systematic review and meta-analysis of the literature, the aim is to determine which monitoring variables would be the strongest predictors of acute (immediately) and residual (up to $72 \mathrm{~h}$ ) fatigue states in soccer. PubMed, SPORTDiscus, and Web of Science databases were searched (until September 2018). Studies concurrently examining soccer match-related external load metrics and subjective and/or objective measures were selected to determine pooled correlations $(\bar{r})$ with confidence intervals $(\mathrm{Cl})$. The quality and strength of the findings of each study were evaluated to identify overall levels of evidence.

Results: Eleven studies were included ( $n=165$ athletes). Acute $(\bar{r}=0.67 ; 95 \% \mathrm{Cl}=[0.40,0.94])$ and residual $(24 \mathrm{~h}$ post-match, $\bar{r}=0.54 ; 95 \% \mathrm{Cl}=[0.35,0.65])$ changes in muscle damage markers and countermovement jump peak power output $(\mathrm{CMJ}$ Ppo $)$ were, with moderate to strong evidence, largely correlated with running distance above 5.5 $\mathrm{m} \mathrm{s}^{-1}$. No other external load metric was largely correlated with both biochemical and neuromuscular markers. For every $100-\mathrm{m}$ run above $5.5 \mathrm{~m} \cdot \mathrm{s}^{-1}$, CK activity measured $24 \mathrm{~h}$ post-match increased by $30 \%$ and $C \mathrm{MJPO}_{\text {PPO }}$ decreased by $0.5 \%$. Conversely, the total distance covered did not present any evidence of a clear relationship with any fatigue-related marker at any time-point.

Conclusions: Running distance above $5.5 \mathrm{~m} \cdot \mathrm{s}^{-1}$ represents the most sensitive monitoring variable characterizing biochemical and neuromuscular responses, at least when assessed during the initial $24 \mathrm{~h}$ (not at $48 \mathrm{~h} / 72 \mathrm{~h}$ ) postmatch recovery period. In addition, total distance covered is not sensitive enough to inform decision-making during the fatigue monitoring process.
\end{abstract}

Keywords: External load, Time motion analysis, Monitoring, Fatigue, Recovery, Muscle damage, Perceptual responses, Neuromuscular performance

\footnotetext{
*Correspondence: joao.silva@aspetar.com; jm_silv@hotmail.com

${ }^{1}$ National Sports Medicine Programme, Excellence in Football Project, Aspetar

- Qatar Orthopaedic and Sports Medicine Hospital, P.O BOX 29222 Doha,

Qatar

${ }^{8}$ Center of Research, Education, Innovation and Intervention in Sport (CIFI2D),

Porto, Portugal

Full list of author information is available at the end of the article
} 


\section{Key Points}

- The running distance covered above $5.5 \mathrm{~m} \cdot \mathrm{s}^{-1}$ represents the most sensitive monitoring variable estimating post-match (up to $+24 \mathrm{~h}$ but not at 48 $72 \mathrm{~h}$ ) changes in biochemical and neuromuscular responses.

- Total distance covered may represent the less sensitive variable to monitor.

- For every $100-\mathrm{m}$ run above $5.5 \mathrm{~m} \cdot \mathrm{s}^{-1}$ during matchplay, creatine kinase activity measured $24 \mathrm{~h}$ postmatch may increase by $30 \%$ and $\mathrm{CMJ}$ peak power output decrease by $0.5 \%$.

\section{Background}

Soccer is considered a high-intensity intermittent sport with an unprecedented increase (up to 50\%) in highimpulsive actions (e.g., number of high accelerations, sprint distance covered) occurring during match-play reported over the last decade [1]. Monitoring players' responses (e.g., physiological and perceptual) to a soccer match is paramount to prescribe the optimal training dose at an individual level, minimize injuries, and restore physical performance for subsequent training and competition $[2,3]$. During the past decade, there has been a substantial development of computer-aided tracking technology (e.g., multiple camera semi-automatic systems) for the examination of players' external load [4] (i.e., activity performed such as total distance covered or the number of accelerations [5]) during training and match-play. These sophisticated systems are now capable of providing detailed analysis of external load demands, which allows individualized performance profiling of players to tailor training programs [4]. The emergence of manufacturer-specific algorithms has prompted the development of some recent parameters (e.g., player load), expressed in absolute or relative terms, yet with a lack of validity and reliability for many of them such as metabolic power and its derivatives [6, 7]. Despite substantial technological advancements, there is a lack of consensus for selecting the most appropriate parameters for quantifying the short-term dose-response relationship and precisely informing on the "stress" experience by each individual player in elite soccer [8].

Soccer match-play is a stressor for various physiological (e.g., musculoskeletal, immunological, metabolic) regulatory systems $[9,10]$. This stress results in acute (i.e., less than $3 \mathrm{~h}$ post-match) and residual (still evident up to $72 \mathrm{~h}$ post-match) fatigue-induced impairments commonly characterized by neuro-mechanical alterations (e.g., decrease in maximal force production capacity) $[10-12]$, perturbations in the biochemical milieu (e.g., increase in creatine kinase levels) [10, 13, 14] and in the psychometric state $[10,15]$. While several factors (e.g., genotype and phenotype) [16] likely influence internal load experienced by each individual player, specific external load metrics (e.g., acceleration variables) may estimate match-related players' fatigue status. It is thought that locomotor activities requiring repeated eccentric muscle contractions (e.g., acceleration and deceleration patterns, high-speed running distance) [17] can explain the aforementioned metabolic, physical, and psychometric disturbances $[13,15,18,19]$ and, in turn, the potential injury causation $[19,20]$. However, the relationship between match external load metrics and markers of post-soccer-match fatigue remains unclear with conflicting results in the literature. For example, the extent of acute muscle damage was correlated with high-intensity (HI) running distance $\left(>4 \mathrm{~m} \mathrm{~s}^{-1}\right)$ with either small $(r=0.24)$ [21] or very large magnitudes $(r=$ 0.92 )[22]. Similar differences in magnitude exist between the extent of residual muscle damage (i.e., $48 \mathrm{~h}$ following the match) and high decelerations $(r=0.19$ and 0.71$)[12,23]$. Therefore, the lack of consensus regarding the effectiveness/preferred external load metrics to monitor players' physiological and biochemical responses to a soccer match implies a need for a systematic review with meta-analysis. This may identify the most sensitive monitoring variables associated with postmatch acute and residual fatigue-related markers. This information may allow informed decisions from a fatigue monitoring standpoint as well from a return to play and performance management perspective.

Therefore, using a systematic review and meta-analysis of the literature, the aim is to determine which external load metrics during a soccer match-play more effectively reflect the acute and residual changes in post-match muscle damage and neuromuscular and perceptual responses.

\section{Methods \\ Literature Search Strategy}

The systematic review with meta-analysis was conducted in accordance with the recommendations defined in the Preferred Reporting Items for Systematic Reviews and Meta-Analyses statement (PRISMA) and the PopulationIntervention-Comparators-Outcomes-Study design (PICOS) approach [24]. The literature search was computerized using PubMed, SPORTDiscus, and Web of Science databases, until the end of September 2018. The complete Boolean search strategy is presented in Table 1. No sex restriction was imposed during the search stage. The reference lists of all articles were examined to identify further eligible studies. Papers published in the epub ahead of print within the abovementioned time frame were also considered.

The PICOS approach of this investigation can be detailed as follows: Population: soccer players. Intervention: official 
Table 1 Database search strategy

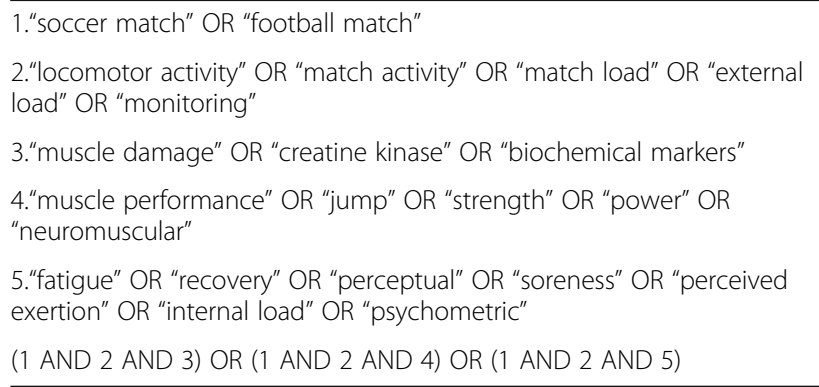

or friendly soccer match without extra time. Comparators: players' soccer match-related external load metrics (e.g., players' running distance above speed thresholds). Outcomes variables: the dependent variables are the acute (i.e., immediately) and residual (1,2, and 3 days following the match) changes in biochemical, neuromuscular, and perceptual measures $[12,25,26]$. Study design: observational studies with a before-after intervention (i.e., a soccer match).

\section{Study Selection and Quality Assessment}

A second phase consisted of applying selected inclusion criteria. These inclusion criteria were as follows:

1. The study was an original research, published in the English language in a peer-review journal.

2. The study population was soccer players.

3. The intervention was an on-field soccer match.

4. The time-motion analysis of locomotor-related activities was reported.

5. Measures of post-match muscular performance or markers of muscle damage or psychometric state were presented.

6. A correlation coefficient reflecting the relationship between one or more external load metrics and post-match fatigue-related markers was reported, or information needed to compute this coefficient was mentioned or available on a supplement file or was obtained from the author(s) of the study.

Attempts were made to contact the authors of the selected articles to request missing data. All authors were given 3 weeks to provide that data. After this period, the studies were assessed for risk of bias using an adapted version of a published scoring system [27]. Ten criteria were determined using the National Heart Blood Institute $(\mathrm{NIH})$ guidelines for qualitative evaluation of observational cohort and cross-sectional studies and beforeafter (pre-post) studies with no control group. In addition, other versions of currently established scales used in sports sciences (e.g., Delphi and PEDro Scale, Newcastle - Ottawa quality assessment scale, Downs and Black) were considered. The quality assessment was based on the reporting of study methods and results with answer categories being "yes," "partial," and "no" (Table 2).

Summary scores (ranging from 0 to 1 ) were calculated as follows:

Summary score $=[($ number of 'yes' $\times 2)+($ number of 'partial' $\times 1)] /($ number of criteria $\times 2)(1)$

Studies were then classified as high $(\geq 0.75)$, moderate $(0.50-0.75)$, or poor methodological quality $(<0.50)$ [27].

\section{Independent Variables}

The independent variables consisted of external load metrics related to soccer match-play. They are presently described by Gray's classification [2] using three distinct levels:

- Level 1: Typical distances covered in different running speeds

- Level 2: All events related to changes in running speed: accelerations, decelerations, and changes of directions

- Level 3: All events derived from the inertial sensors/ accelerometers such as impacts above gravitational force thresholds

However, in each individual selected study, external load variables may have been presented with specific speed thresholds and, in turn, defined differently. Sprinting pattern, for example, has been defined as running speed above different thresholds: (i) $5 \mathrm{~m} \mathrm{~s}^{-1}$ [22], (ii) 5.5 $\mathrm{m} \mathrm{s}^{-1}$ [12], (iii) $5.8 \mathrm{~m} \mathrm{~s}^{-1}$ [23], and (iv) $7 \mathrm{~m} \mathrm{~s}^{-1}$ [21]. Consequently, the independent variables were grouped by common zones based on thresholds used by practitioners on the field in elite soccer [8]:

- High-intensity running (HIR): running speed greater than $\sim 4 \mathrm{~m} \mathrm{~s}^{-1}$.

- Very high-intensity running (VHIR): running speed greater than 5 to $5.5 \mathrm{~m} \mathrm{~s}^{-1}$

- Sprint: running speed greater than $7 \mathrm{~m} \mathrm{~s}^{-1}$.

- Moderate to high-intensity acceleration: acceleration greater or equal to $+2 \mathrm{~m} \cdot \mathrm{s}^{-2}$.

- High-intensity acceleration: acceleration greater or equal to $+3 \mathrm{~m} \mathrm{~s}^{-2}$.

- High-intensity deceleration: a deceleration lower or equal to $-3 \mathrm{~m} \mathrm{~s}^{-2}$.

- Moderate- to high-intensity deceleration: a deceleration lower or equal to $-2 \mathrm{~m} \mathrm{~s}^{-2}$.

- High-intensity impact: impact greater than $7 \mathrm{G}$ (gravitational force). The impact is the instantaneous rate of acceleration and deceleration in the three axes, measured by the integrated accelerometer. 
Table 2 Quality assessment criteria

\begin{tabular}{|c|c|c|c|c|}
\hline \multirow[t]{2}{*}{ No. } & \multirow[t]{2}{*}{ Items } & \multicolumn{3}{|c|}{ Scoring } \\
\hline & & 0 & 1 & 2 \\
\hline 1 & Was the main question or objective clearly described? & No & & \\
\hline 2 & Was the study population clearly specified and defined (age, gender, training status, stated inclusion/exclusion criteria)? & No & Partly & \\
\hline 3 & Was the sample size justified? & No & & \\
\hline 4 & Main measured variables clearly described in the Introduction or Method section? & No & Partly & \\
\hline 5 & Locomotor activity variables clearly defined (thresholds, ranges). & No & Partly & \\
\hline 6 & Were the validity and reliability of the main variables measurements discussed? & No & Partly & \\
\hline 7 & Were the methods (included the statistical methods) sufficiently described to enable the study replication? & No & Partly & \\
\hline 8 & $\begin{array}{l}\text { Does the study provide estimates of the random variability in the data for the main outcomes (i.e. confidence intervals, } \\
\text { standard deviations)? }\end{array}$ & No & Partly & \\
\hline 9 & Were all the tested associations reported? & No & & \\
\hline 10 & Were the study limitations discussed? & No & Partly & \\
\hline
\end{tabular}

- High change of direction: change of direction with a high-intensity deceleration.

\section{Dependent Variables}

The dependent variables extracted from the selected studies were systematically reviewed and grouped into three categories: biochemical, neuromuscular, and perceptual measures. Changes in the biochemical milieu were assessed through endocrine, immunological, and muscle damage markers (Table 3). Endocrine alterations consisted of changes in testosterone and cortisol concentrations. Changes in immunological markers were assessed by leucocytes counts. Changes in muscle damage markers consisted of measures of intracellular enzyme activity (creatine kinase and lactate dehydrogenase, $\mathrm{CK}$ and LDH, respectively) and in circulating concentrations of Myoglobin (Mb). The neuromuscular function was assessed by (i) maximal voluntary contractions using isokinetic dynamometers to measure several forcerelated variables (e.g., peak torque achieved by different lower-limbs muscle groups under maximal isometric; MVIC), concentric and eccentric contractions, (ii) vertical jump performance using force plates and/or optical timing systems (e.g., jump peak power output during a countermovement, $\mathrm{CMJ}_{\mathrm{PPO}}$ ). The perceptual responses were mainly based on lower limb delayed onset of muscle soreness (DOMS), rate of perceived exertion $(\mathrm{RPE})$, the perceived recovery (TQR), and the brief assessment of mood (BAM+) measures. DOMS was assessed with visual analog scales in response or not to a "conditioning" stimulus (e.g., squatting). Other scales were used to quantify RPE, TQR, and BAM+.

\section{Analysis and Interpretation of Results}

To determine the relationship between match-related external load variables and post-match fatigue-related measurements, each relationship from an individual study was rated according to its direction (positive, negative, no relationship) and its magnitude as determined from reported correlations. The criteria adopted to categorize magnitudes of correlations $(r)$ were as follows: $\leq 0.1$, trivial; > 0.1-0.3, small; > 0.3-0.5, moderate; > $0.5-0.7$, large; > 0.7-0.9, very large; and > 0.9-1.0, almost perfect [34]. Individual relationships were then summed and rated according to the predetermined levels of evidence adapted from Van Tulder et al. [35] recommendations:

$>$ Strong evidence: consistently identified in two or more studies, which presented low heterogeneity $\left(I^{2}<\right.$ $30 \%$ ) and including a minimum of two high-quality studies.

$\triangleright$ Moderate evidence: consistently identified in two or more studies, which presented low heterogeneity $\left(I^{2}<\right.$ $30 \%)$ and including at least one high-quality study.

$\triangleright$ Limited evidence: identified in one high-quality study, or multiple low- to moderate-quality studies that may not present low heterogeneity $\left(I^{2}<30 \%\right)$.

$\triangleright$ Conflicting evidence: inconsistency in two or more studies where half of the studies are in agreement and the other half conflicting.

$\triangleright$ No evidence: pooled results that are insignificant and derived from multiple moderately to substantially heterogeneous studies $\left(I^{2}>30 \%\right)$.

"Inconsistency" refers to a lack of similarity for correlation coefficients across studies. Study results are considered consistent when direction, magnitude, and statistical significance are sufficiently similar to lead to the same conclusions [36]. Consistency in direction is defined as $75 \%$ or more of the studies showing either a positive or negative correlation. Consistency in magnitude is defined as $75 \%$ or more of the studies showing an important or unimportant relationship [36]. Heterogeneity between studies was assessed using the $I^{2}$ statistic, where an $I^{2}$ of $30 \%$ or less is considered to indicate 
Table $\mathbf{3}$ Characteristics of the selected studies

\begin{tabular}{|c|c|c|c|c|c|}
\hline Studies & $\begin{array}{l}\text { Players } \\
\text { details (level, } \\
n, \text { age) }\end{array}$ & $\begin{array}{l}\text { Tracking } \\
\text { systems }\end{array}$ & External load metrics examined & $\begin{array}{l}\text { Fatigue-related dependent variables } \\
\text { investigated }\end{array}$ & $\begin{array}{l}\text { Quality } \\
\text { assessment } \\
\text { score }\end{array}$ \\
\hline $\begin{array}{l}\text { Aquino } \\
\text { et al., [28] }\end{array}$ & $\begin{array}{l}\text { Elite/18/15.6 } \\
\pm 0.4\end{array}$ & $\begin{array}{l}\text { Video analysis } \\
\text { "tracking } \\
\text { software } \\
\text { DVIDEOW" }\end{array}$ & $\begin{array}{l}\text { Medium running distance }\left(8.1-13 \mathrm{~km} \mathrm{~h}^{-1}\right) \text {; HI } \\
\text { running distance }\left(13.1-18 \mathrm{~km} \mathrm{~h}^{-1}\right) \text {; Sprinting } \\
\text { distance }\left(>18 \mathrm{~km} \mathrm{~h}^{-1}\right) ; \mathrm{H} \text { activity distance }(> \\
\left.13 \mathrm{~km} \mathrm{~h}^{-1}\right) ; \mathrm{TD} ; \text { Number of sprints. }\end{array}$ & $\begin{array}{l}\text { Pre- to post-match \% change in CK } \\
\text { concentration } \\
\text { Pre- to post-match \% change in LDH } \\
\text { concentration }\end{array}$ & 0.65 \\
\hline $\begin{array}{l}\text { De Hoyo } \\
\text { et al., [23] }\end{array}$ & $\begin{array}{l}\text { Elite/15/18 } \\
\pm 1\end{array}$ & $\begin{array}{l}\text { 15- } \mathrm{Hz} \text { GPS with } \\
100-\mathrm{Hz} \\
\text { accelerometer }\end{array}$ & $\begin{array}{l}\text { Medium intensity }\left(14-17.9 \mathrm{~km} \mathrm{~h}^{-1}\right) \text {, HI }(18-20.9 \\
\left.\mathrm{km} \mathrm{h} \mathrm{h}^{-1}\right) \text { and sprinting }\left(>21 \mathrm{~km} \mathrm{~h} \mathrm{~h}^{-1}\right) \text { distances; } \\
\text { High-speed }\left(>14 \mathrm{~km} \mathrm{~h}^{-1}\right) \text { and very high-speed } \\
\text { running }\left(>18 \mathrm{~km} \mathrm{~h}^{-1}\right) \text {; number of medium to } \\
\text { high }\left(>2 \mathrm{~m} / \mathrm{s}^{2}\right) \text { and high }\left(>3 \mathrm{~m} / \mathrm{s}^{2}\right) \text { accelera- } \\
\text { tions; number of medium to high }\left(<-2 \mathrm{~m} / \mathrm{s}^{2}\right) \\
\text { and high decelerations }\left(<-3 \mathrm{~m} / \mathrm{s}^{2}\right) \text {; TD; } 1 \mathrm{~m}- \\
\text { pacts }(>7.1 \mathrm{G})\end{array}$ & $\begin{array}{l}\text { Pre to post-match changes at } \sim 30 \\
\text { min, } \mathrm{G}+24 \mathrm{H} \text { and } \mathrm{G}+48 \mathrm{H} \text { in: } \\
\mathrm{CK} \text { concentration } \\
\text { Average concentric and eccentric force } \\
\text { during CMJ } \\
\mathrm{CMJ} \text { height }\end{array}$ & 0.70 \\
\hline $\begin{array}{l}\text { Draganidis } \\
\text { et al., [21] }\end{array}$ & $\begin{array}{l}\text { Semi- } \\
\text { professional/ } \\
20 / 22.6 \pm \\
1.5\end{array}$ & $\begin{array}{l}\text { 5- } \mathrm{Hz} \text { GPS with } \\
100-\mathrm{Hz} \\
\text { accelerometer }\end{array}$ & $\begin{array}{l}\text { HI running }\left(>14.5 \mathrm{~km} \mathrm{~h}^{-1}\right) \text {; VHI running }(>19.8 \\
\left.\mathrm{km} \mathrm{h} \mathrm{h}^{-1}\right) \text { and sprinting }\left(>25.2 \mathrm{~km} \mathrm{~h} \mathrm{~h}^{-1}\right) \text {; number } \\
\text { of low, medium and } \mathrm{HI} \text { accelerations }\left(1-2 \mathrm{~m} / \mathrm{s}^{2} \text {, }\right. \\
\left.2-3 \mathrm{~m} / \mathrm{s}^{2},>3 \mathrm{~m} / \mathrm{s}^{2}\right) \text {; Number of low, medium } \\
\text { and } \mathrm{HI} \text { decelerations }\left(-1 \text { to }-2 \mathrm{~m} / \mathrm{s}^{2},-2 \text { to }-\right. \\
\left.3 \mathrm{~m} / \mathrm{s}^{2},<-3 \mathrm{~m} / \mathrm{s}^{2}\right) \text {; TD }\end{array}$ & $\begin{array}{l}\text { Pre to } \mathrm{G}+24 \mathrm{H}, \mathrm{G}+48 \mathrm{H} \text { and } \mathrm{G}+72 \mathrm{H} \\
\text { changes in: } \\
\text { CK concentration } \\
\text { Leucocytes count } \\
\text { Concentric and eccentric isokinetic } \\
\text { peak torque at } 60^{\circ} / \mathrm{s} \text { and } 180^{\circ} / \mathrm{s} \\
\mathrm{MVIC} \text { of } \mathrm{KE} \text { and } \mathrm{KF} \text { muscles } \\
\text { DOMS }\end{array}$ & 0.80 \\
\hline $\begin{array}{l}\text { Nedelec } \\
\text { et al., [25] }\end{array}$ & $\begin{array}{l}\text { Professional/ } \\
14 / 21.8 \pm \\
3.2\end{array}$ & Video analysis & $\begin{array}{l}\text { High acceleration and deceleration }<5 \mathrm{~m} \\
\text { High acceleration and deceleration }>5 \mathrm{~m} \\
\mathrm{HI} \text { running } \\
\text { Hard changes of direction }\end{array}$ & $\begin{array}{l}\text { Pre to } \mathrm{G}+24 \mathrm{H}, \mathrm{G}+48 \mathrm{H} \text { and } \mathrm{G}+72 \mathrm{H} \\
\text { post-match changes in: } \mathrm{CMJ} \text { perform- } \\
\text { ance } \mathrm{MVIC} \text { of the hamstring with knee } \\
\text { flexed at } 90^{\circ} \text { and } 150^{\circ} \mathrm{KF} \text { muscle } \\
\text { soreness }\end{array}$ & 0.65 \\
\hline $\begin{array}{l}\text { Rampinini } \\
\text { et al., [29] }\end{array}$ & $\begin{array}{l}\text { Professional/ } \\
20 / 19 \pm 1\end{array}$ & $\begin{array}{l}\text { Video- } \\
\text { computerized, } \\
\text { semi-automatic } \\
\text { match analysis }\end{array}$ & $\begin{array}{l}\text { TD } \\
\text { HI running }\left(>15 \mathrm{~km} \mathrm{~h}^{-1}\right)\end{array}$ & $\begin{array}{l}\text { Pre to immediately, G }+24 \mathrm{H} \text { and } \mathrm{G}+ \\
48 \mathrm{H} \% \text { changes in: } \\
\text { Maximal voluntary activation (\%VA) } \\
\text { Normalized Root mean square of } \\
\text { vastus lateralis muscle EMG signal } \\
\text { during MVIC. } \\
\text { Peak torque with 1-ms, 10-ms interval } \\
\text { and 100-ms interval stimulations }\end{array}$ & 0.70 \\
\hline $\begin{array}{l}\text { Romagnoli } \\
\text { et al., [30] }\end{array}$ & $\begin{array}{l}\text { Professional/ } \\
20 / 17-20\end{array}$ & $\begin{array}{l}\text { Video- } \\
\text { computerized, } \\
\text { semi-automatic } \\
\text { match analysis }\end{array}$ & $\begin{array}{l}\text { TD } \\
\text { Low-intensity running distance }\left(<15 \mathrm{~km} \mathrm{~h}^{-1}\right) \\
\text { HI running distance }\left(>15 \mathrm{~km} \mathrm{~h}^{-1}\right) \\
\text { VHI running distance }\left(>20 \mathrm{~km} \mathrm{~h}^{-1}\right)\end{array}$ & $\begin{array}{l}\text { Pre to } 30 \mathrm{~min}, \mathrm{G}+24 \mathrm{H} \text { and } \mathrm{G}+48 \mathrm{H} \\
\text { changes in: } \\
\text { White blood cells count } \\
\text { Cortisol and Testosterone } \\
\text { concentrations } \\
\text { Creatine kinase concentration }\end{array}$ & 0.40 \\
\hline $\begin{array}{l}\text { Russell } \\
\text { et al., [12] }\end{array}$ & $\begin{array}{l}\text { Professional/ } \\
15 / \\
20 \pm 1\end{array}$ & 10-Hz GPS units & $\begin{array}{l}\text { Raw and normalized (per min) TD } \\
\text { Raw and normalized (per min) HI running } \\
\text { distance }\left(>19.8 \mathrm{~km} \mathrm{~h}^{-1} \text { ) }\right. \\
\text { Raw and normalized (per min) total number of: } \\
\text { sprints }\left(>19.8 \mathrm{~km} \mathrm{~h}^{-1}\right) \text {, high accelerations, high } \\
\text { decelerations, } \mathrm{HI} \text { impacts } \\
\text { Total number of accelerations, decelerations } \\
\text { and impacts. }\end{array}$ & $\begin{array}{l}\text { Pre to } \mathrm{G}+24 \mathrm{H} \text { and } \mathrm{G}+48 \mathrm{H} \text { changes } \\
\text { in: CK concentration } \\
\text { CMJ PPO }\end{array}$ & 0.80 \\
\hline $\begin{array}{l}\text { Scott et al., } \\
\text { [31] }\end{array}$ & $\begin{array}{l}\text { Professional/ } \\
15 / 26 \pm 4\end{array}$ & $\begin{array}{l}\text { video- } \\
\text { computerized, } \\
\text { semi-automatic } \\
\text { match analysis }\end{array}$ & $\begin{array}{l}\left.\text { TD, HI running (> } 19.8 \mathrm{~km} \mathrm{~h}^{-1}\right) \text { and Sprinting (> } \\
\left.25.2 \mathrm{~km} \mathrm{~h}^{-1}\right) \text { : distances and occurrences }\end{array}$ & $\begin{array}{l}\text { Pre to } \mathrm{G}+48 \mathrm{H} \text { changes in } \mathrm{CK} \\
\text { concentration }\end{array}$ & 0.75 \\
\hline $\begin{array}{l}\text { Shearer } \\
\text { et al., [32] }\end{array}$ & $\begin{array}{l}\text { Elite/11/20 } \\
\pm 1\end{array}$ & 10-Hz GPS units & $\begin{array}{l}\text { Raw and normalized (per min) TD } \\
\text { Raw and normalized (per min) HI running } \\
\text { distance }\left(>19.8 \mathrm{~km} \mathrm{~h}^{-1}\right) \\
\text { Raw and normalized (per min) total number of } \\
\text { sprints }\left(>19.8 \mathrm{~km} \mathrm{~h}^{-1} \text { ) }\right.\end{array}$ & $\begin{array}{l}\text { Pre to } \mathrm{G}+24 \mathrm{H} \text { and } \mathrm{G}+48 \mathrm{H} \text { changes } \\
\text { in: } \\
\text { CK concentration } \\
\text { CMJ PPO } \\
\text { Brief assessment of mood }\end{array}$ & 0.75 \\
\hline $\begin{array}{l}\text { Thorpe } \\
\text { et al., [22] }\end{array}$ & $\begin{array}{l}\text { Semi- } \\
\text { professional/ } \\
7 / 25 \pm 6\end{array}$ & $\begin{array}{l}\text { 1-Hz GPS unit } \\
\text { with } 100-\mathrm{Hz} \\
\text { accelerometer }\end{array}$ & $\begin{array}{l}\text { Walking distance }\left(0-6 \mathrm{~km} \mathrm{~h}^{-1}\right) \text {; jogging distance } \\
\left(6-8 \mathrm{~km} \mathrm{~h}^{-1}\right) \text {; low-speed running }\left(8-12 \mathrm{~km} \mathrm{~h}^{-1}\right) \text {; } \\
\text { moderate-speed running }\left(12-15 \mathrm{~km} \mathrm{~h}^{-1}\right) \text {; Fast } \\
\text { speed running }\left(15-18 \mathrm{~km} \mathrm{~h}^{-1}\right) \text {; sprinting dis- } \\
\text { tance }\left(>18 \mathrm{~km} \mathrm{~h}^{-1}\right) \text {; Hl activity }\left(>15 \mathrm{~km} \mathrm{~h}^{-1}\right) \text {; }\end{array}$ & $\begin{array}{l}\text { Pre to post-match changes in: } \\
\text { CK concentration } \\
\text { Myoglobin concentration } \\
\text { Cortisol and Testosterone } \\
\text { concentrations }\end{array}$ & 0.70 \\
\hline
\end{tabular}


Table 3 Characteristics of the selected studies (Continued)

\begin{tabular}{|c|c|c|c|c|c|}
\hline Studies & $\begin{array}{l}\text { Players } \\
\text { details (level, } \\
n \text {, age) }\end{array}$ & $\begin{array}{l}\text { Tracking } \\
\text { systems }\end{array}$ & External load metrics examined & $\begin{array}{l}\text { Fatigue-related dependent variables } \\
\text { investigated }\end{array}$ & $\begin{array}{l}\text { Quality } \\
\text { assessment } \\
\text { score }\end{array}$ \\
\hline & & & total number of sprints. & & \\
\hline $\begin{array}{l}\text { Varley } \\
\text { et al., [33] }\end{array}$ & $\begin{array}{l}\text { Professional/ } \\
10 / 27 \pm 3\end{array}$ & $\begin{array}{l}\text { Video- } \\
\text { computerized, } \\
\text { semi-automatic } \\
\text { match analysis }\end{array}$ & $\begin{array}{l}\left.\text { TD, sprint distance (> } 25.2 \mathrm{~km} \mathrm{~h}^{-1}\right) \text {, total } \\
\text { number of: sprints), high accelerations, high } \\
\text { decelerations. }\end{array}$ & $\begin{array}{l}\text { Pre to immediately, } \mathrm{G}+48 \mathrm{H} \text { and } \mathrm{G}+ \\
72 \mathrm{H} \text { changes in: } \\
\mathrm{CK} \text { concentration } \\
\mathrm{CMJ} \text { height } \\
\text { Muscle fatigue and wellness }\end{array}$ & 0.85 \\
\hline
\end{tabular}

$n$ : number of players, TD: total distance, KE: knee extension, KF: knee flexion, CMJ: countermovement jump, PPO: peak power output, PT: peak torque, DOMS: delayed onset muscle soreness, $\mathrm{HI}$ : high intensity, VHI: very high intensity, MVIC: maximal voluntary isometric contraction

low heterogeneity and the cutoffs of $30 \%<\mathrm{I}^{2}<50 \%$ and $I^{2}>50 \%$ are indicative of moderate and substantial heterogeneity, respectively [37]. $I^{2}$ describes the percentage of total variation across studies that is due to heterogeneity rather than chance and seeks to determine whether there are genuine differences underlying the results of the studies (heterogeneity), or whether the variation in findings is compatible with chance alone (homogeneity) [37]. Heterogeneity between studies was also assessed by the chi-squared test [36].

The meta-analysis was processed in two consecutive phases. Some studies investigating the changes in muscle damage determined the activity of different serum skeletal muscle proteins (e.g., CK and LDH). As various markers are believed to provide a composite picture of muscle damage status, using more than one marker has been recommended [38]. Consequently, the correlation coefficients, associating two muscle damage markers (e.g., CK and LDH) with identical external load metric (e.g., HI running distance) were combined in this first step to obtain a single within-study correlation coefficient. The specific relationships (e.g., HI running distance vs. muscle damage markers) assessed in several selected studies were then meta-analyzed between studies during the second phase.

The meta-analytic procedure was conducted with StatsDirect software (v 2.8.0, StatsDirect, Cheshire, UK) package allowing to calculate pooled correlation coefficients $(\bar{r})$ with three different methods: (i) Hedges and Olkins random-effects method [39], (ii) Hedges and Olkins fixed-effects method and, (iii) Hunter and Schmidt random-effects method [40]. The latter method systematically presented equivalent or the lowest pooled correlation coefficient. Therefore, to minimize overestimation bias, it was decided to consider the results from Hunter and Schmidt method only (Tables 4 and 5 ). Moreover, for either a small number of studies (less than 30) or a heterogeneous set of studies, the least biased estimate of the true population correlation is believed to be provided by Hunter and Schmidt method [41]. Pooled correlation coefficients are presented with $95 \%$ of confidence limits/intervals $(\mathrm{CL} / \mathrm{CI})$. Finally, once the strongest predictor has been determined, the studies investigating this external variable were selected and the authors were contacted to obtain individual data. This allowed us to provide a relationship between this predictor and fatigue-related markers (percentage change in $\mathrm{CMJ}_{\mathrm{PPO}}$ and CK, Fig. 2).

\section{Results}

\section{Study and Data Characteristics}

The flow chart of the search and selection process is presented in Fig. 1. In summary, the searches identified 1456 relevant articles including all reference lists. After critically analyzing the titles and abstracts, the total number of relevant articles was reduced to 551. Once applying the selection criteria, eleven cohort studies were selected (Fig. 1) [12, 22, 23, 25, 36-42] and data were extracted for meta-analysis. The total number of players was 165 with $89 \%$ belonging to the elite level and only male soccer players were finally represented (Table 3 ). The average methodological quality of the included articles was $0.70 \pm 0.12$ (mean \pm standard deviation) out of 1 , ranging from 0.40 to 0.85 , with 4 articles considered of a high $(\geq 0.75)$ methodological quality (Table 3$)$.

Eight main external load variables with 10 additional derivatives were used in the selected studies as follows:

- Level 1: Total distance (TD, 8 studies), HIR (7 studies), VHIR (7 studies), sprints (3 studies)

- Level 2: Accelerations (5 studies) and decelerations (5 studies), high change of directions (1 study).

- Level 3: High impacts (2 studies)

The most common group of dependent variables measured in the selected studies was the muscle damage biochemical markers (8 studies). The other dependent variables represented in at least two studies were CMJ peak power output $\left(\mathrm{CMJ}_{\mathrm{PPO}}, 4\right.$ studies), MVIC (2 studies) and DOMS (2 studies)

These variables were measured within the first $3 \mathrm{~h}$ (Post; 6 studies), one ( $\mathrm{G}+24 \mathrm{H} 7$ studies), two ( $\mathrm{G}+$ $48 \mathrm{H} ; 8$ studies $)$ and three days $(\mathrm{G}+72 \mathrm{H} ; 3$ studies $)$ post-match. 
Table 4 Summary of findings from meta-analysis describing the relationship between external load metrics of level 1 [3] and fatigue-related markers

\begin{tabular}{|c|c|c|c|c|c|c|c|c|c|c|c|}
\hline Predictors & Fatigue-related markers & Time & $\bar{r}$ & Lower CL & Upper CL & Number of studies/correlations & Evidence & $n$ & $P^{2}$ & $\mathrm{chi}^{2}$ & $p$ \\
\hline \multirow[t]{37}{*}{$\mathrm{TD}$} & \multirow[t]{4}{*}{ Muscle damage } & Post & 0.36 & 0.05 & 0.67 & $5 / 3$ & Limited & 48 & 66 & 2.95 & 0.09 \\
\hline & & $\mathrm{G}+24 \mathrm{H}$ & 0.36 & 0.19 & 0.53 & $5 / 6$ & Limited & 87 & 0 & 4.73 & 0.45 \\
\hline & & $\mathrm{G}+48 \mathrm{H}$ & 0.23 & 0.05 & 0.42 & $6 / 7$ & Moderate & 97 & 0 & 4.81 & 0.44 \\
\hline & & $\mathrm{G}+72 \mathrm{H}$ & -0.02 & & & $1 / 1$ & No & 10 & & & \\
\hline & \multirow[t]{2}{*}{$C M J_{P P O}$} & $\mathrm{G}+24 \mathrm{H}$ & -0.16 & -0.47 & 0.15 & $2 / 5$ & Conflicted & 67 & 41 & 7.83 & 0.10 \\
\hline & & $\mathrm{G}+48 \mathrm{H}$ & 0.09 & -0.08 & 0.27 & $2 / 5$ & Moderate & 67 & 0 & 2.46 & 0.65 \\
\hline & \multirow[t]{2}{*}{ CMJ performance } & $\mathrm{G}+48 \mathrm{H}$ & 0.04 & & & $1 / 1$ & No & 10 & & & \\
\hline & & $\mathrm{G}+72 \mathrm{H}$ & -0.06 & & & $1 / 1$ & No & 10 & & & \\
\hline & \multirow[t]{3}{*}{ Leucocytes count } & $\mathrm{G}+24 \mathrm{H}$ & -0.10 & & & $2 / 1$ & No & 39 & & & \\
\hline & & $\mathrm{G}+48 \mathrm{H}$ & 0.21 & & & $2 / 1$ & No & 39 & & & \\
\hline & & $\mathrm{G}+72 \mathrm{H}$ & 0.09 & & & $2 / 1$ & No & 39 & & & \\
\hline & \multirow[t]{3}{*}{ Cortisol } & Post & NS & & & $1 / 0$ & No & & & & \\
\hline & & $\mathrm{G}+24 \mathrm{H}$ & 0.50 & & & $1 / 1$ & Limited & 20 & & & \\
\hline & & $\mathrm{G}+48 \mathrm{H}$ & 0.52 & & & $1 / 1$ & Limited & 20 & & & \\
\hline & \multirow[t]{3}{*}{ Testosterone } & Post & NS & & & $1 / 1$ & No & 20 & & & \\
\hline & & $\mathrm{G}+24 \mathrm{H}$ & NS & & & $1 / 1$ & No & 20 & & & \\
\hline & & $\mathrm{G}+48 \mathrm{H}$ & NS & & & $1 / 1$ & No & 20 & & & \\
\hline & PT $1 \mathrm{~Hz}$ & Post & -0.19 & & & $1 / 1$ & No & 19 & & & \\
\hline & PT $10 \mathrm{~Hz}$ & Post & -0.20 & & & $1 / 1$ & No & 19 & & & \\
\hline & PT $100 \mathrm{~Hz}$ & Post & -0.17 & & & $1 / 1$ & No & 19 & & & \\
\hline & \multirow[t]{3}{*}{ DOMS } & $\mathrm{G}+24 \mathrm{H}$ & -0.04 & & & $1 / 1$ & No & 20 & & & \\
\hline & & $\mathrm{G}+48 \mathrm{H}$ & 0.06 & & & $1 / 1$ & No & 20 & & & \\
\hline & & $\mathrm{G}+72 \mathrm{H}$ & 0.04 & & & $1 / 1$ & No & 20 & & & \\
\hline & \multirow[t]{3}{*}{ PT Concentric KE } & $\mathrm{G}+24 \mathrm{H}$ & -0.68 & & & $1 / 1$ & Limited & 20 & & & \\
\hline & & $\mathrm{G}+48 \mathrm{H}$ & 0.43 & & & $1 / 1$ & Limited & 20 & & & \\
\hline & & $\mathrm{G}+72 \mathrm{H}$ & 0.42 & & & $1 / 1$ & Limited & 20 & & & \\
\hline & \multirow[t]{3}{*}{ PT Concentric KF } & $G+24 H$ & -0.76 & & & $1 / 1$ & Limited & 20 & & & \\
\hline & & $\mathrm{G}+48 \mathrm{H}$ & 0.65 & & & $1 / 1$ & Limited & 20 & & & \\
\hline & & $\mathrm{G}+72 \mathrm{H}$ & 0.58 & & & $1 / 1$ & Limited & 20 & & & \\
\hline & \multirow[t]{3}{*}{ PT Eccentric KE } & $\mathrm{G}+24 \mathrm{H}$ & -0.59 & & & $1 / 1$ & Limited & 20 & & & \\
\hline & & $\mathrm{G}+48 \mathrm{H}$ & 0.40 & & & $1 / 1$ & Limited & 20 & & & \\
\hline & & $\mathrm{G}+72 \mathrm{H}$ & 0.44 & & & $1 / 1$ & Limited & 20 & & & \\
\hline & \multirow[t]{3}{*}{ PT Eccentric KF } & $\mathrm{G}+24 \mathrm{H}$ & -0.82 & & & $1 / 1$ & Limited & 20 & & & \\
\hline & & $\mathrm{G}+48 \mathrm{H}$ & 0.58 & & & $1 / 1$ & Limited & 20 & & & \\
\hline & & $\mathrm{G}+72 \mathrm{H}$ & 0.56 & & & $1 / 1$ & Limited & 20 & & & \\
\hline & \multirow[t]{2}{*}{ BAM+ } & $G+24 H$ & 0.09 & & & $1 / 1$ & No & 35 & & & \\
\hline & & $\mathrm{G}+48 \mathrm{H}$ & 0.14 & & & $1 / 1$ & No & 35 & & & \\
\hline \multirow[t]{6}{*}{$\mathrm{TD} / \mathrm{min}$} & \multirow[t]{2}{*}{ Muscle damage } & $\mathrm{G}+24 \mathrm{H}$ & 0.13 & 0.05 & 0.26 & $2 / 2$ & Limited & 67 & & & \\
\hline & & $\mathrm{G}+48 \mathrm{H}$ & -0.06 & 0.00 & -0.11 & $2 / 2$ & Limited & 67 & & & \\
\hline & \multirow[t]{2}{*}{$C M J_{P P O}$} & $\mathrm{G}+24 \mathrm{H}$ & -0.03 & -0.05 & -0.01 & $2 / 2$ & Limited & 67 & & & \\
\hline & & $\mathrm{G}+48 \mathrm{H}$ & -0.11 & -0.11 & -0.10 & $2 / 2$ & Limited & 67 & & & \\
\hline & \multirow[t]{2}{*}{ BAM+ } & $\mathrm{G}+24 \mathrm{H}$ & -0.47 & & & $1 / 1$ & Limited & 35 & & & \\
\hline & & $\mathrm{G}+48 \mathrm{H}$ & -0.51 & & & $1 / 1$ & Limited & 35 & & & \\
\hline
\end{tabular}


Table 4 Summary of findings from meta-analysis describing the relationship between external load metrics of level 1 [3] and fatigue-related markers (Continued)

\begin{tabular}{|c|c|c|c|c|c|c|c|c|c|c|c|}
\hline Predictors & Fatigue-related markers & Time & $\bar{r}$ & Lower CL & Upper CL & Number of studies/correlations & Evidence & $n$ & $P^{2}$ & $\mathrm{chi}^{2}$ & $p$ \\
\hline \multirow[t]{32}{*}{ HIR } & \multirow[t]{3}{*}{ Muscle damage } & Post & 0.55 & 0.33 & 0.78 & $5 / 3$ & Moderate & 45 & 59 & 3.40 & 0.18 \\
\hline & & $\mathrm{G}+24 \mathrm{H}$ & 0.37 & & & $3 / 1$ & Limited & 20 & & & \\
\hline & & $\mathrm{G}+48 \mathrm{H}$ & 0.37 & 0.21 & 0.53 & $3 / 2$ & Limited & 35 & 0 & 0.57 & 0.45 \\
\hline & \multirow[t]{3}{*}{ MVIC Dom } & $\mathrm{G}+24 \mathrm{H}$ & -0.55 & & & $2 / 1$ & Limited & 20 & & & \\
\hline & & $\mathrm{G}+48 \mathrm{H}$ & 0.43 & & & $2 / 1$ & Limited & 20 & & & \\
\hline & & $\mathrm{G}+72 \mathrm{H}$ & 0.46 & & & $2 / 1$ & Limited & 20 & & & \\
\hline & \multirow[t]{3}{*}{ DOMS } & $\mathrm{G}+24 \mathrm{H}$ & -0.03 & & & $2 / 1$ & No & 34 & & & \\
\hline & & $\mathrm{G}+48 \mathrm{H}$ & 0.00 & & & $2 / 1$ & No & 34 & & & \\
\hline & & $\mathrm{G}+72 \mathrm{H}$ & 0.04 & & & $2 / 1$ & No & 34 & & & \\
\hline & \multirow[t]{2}{*}{ Leucocytes count } & $G+24 H$ & -0.11 & & & $2 / 1$ & No & 39 & & & \\
\hline & & $\mathrm{G}+48 \mathrm{H}$ & 0.21 & & & $2 / 1$ & No & 39 & & & \\
\hline & \multirow[t]{3}{*}{ Cortisol } & Post & NS & & & $2 / 0$ & No & & & & \\
\hline & & $\mathrm{G}+24 \mathrm{H}$ & NS & & & $1 / 0$ & No & & & & \\
\hline & & $\mathrm{G}+48 \mathrm{H}$ & NS & & & $1 / 0$ & No & & & & \\
\hline & \multirow[t]{3}{*}{ Testosterone } & Post & NS & & & $2 / 0$ & No & & & & \\
\hline & & $\mathrm{G}+24 \mathrm{H}$ & NS & & & $1 / 0$ & No & & & & \\
\hline & & $\mathrm{G}+48 \mathrm{H}$ & NS & & & $1 / 0$ & No & & & & \\
\hline & PT $1 \mathrm{~Hz}$ & Post & -0.22 & & & $1 / 1$ & No & 19 & & & \\
\hline & PT $10 \mathrm{~Hz}$ & Post & -0.24 & & & $1 / 1$ & No & 19 & & & \\
\hline & PT $100 \mathrm{~Hz}$ & Post & -0.20 & & & $1 / 1$ & No & 19 & & & \\
\hline & \multirow[t]{3}{*}{ PT Concentric KE } & $\mathrm{G}+24 \mathrm{H}$ & -0.69 & & & $1 / 1$ & Limited & 20 & & & \\
\hline & & $\mathrm{G}+48 \mathrm{H}$ & 0.46 & & & $1 / 1$ & Limited & 20 & & & \\
\hline & & $\mathrm{G}+72 \mathrm{H}$ & 0.45 & & & $1 / 1$ & Limited & 20 & & & \\
\hline & \multirow[t]{3}{*}{ PT Concentric KF } & $\mathrm{G}+24 \mathrm{H}$ & -0.77 & & & $1 / 1$ & Limited & 20 & & & \\
\hline & & $\mathrm{G}+48 \mathrm{H}$ & 0.68 & & & $1 / 1$ & Limited & 20 & & & \\
\hline & & $\mathrm{G}+72 \mathrm{H}$ & 0.59 & & & $1 / 1$ & Limited & 20 & & & \\
\hline & \multirow[t]{3}{*}{ PT Eccentric KE } & $\mathrm{G}+24 \mathrm{H}$ & -0.59 & & & $1 / 1$ & Limited & 20 & & & \\
\hline & & $\mathrm{G}+48 \mathrm{H}$ & 0.43 & & & $1 / 1$ & Limited & 20 & & & \\
\hline & & $\mathrm{G}+72 \mathrm{H}$ & 0.46 & & & $1 / 1$ & Limited & 20 & & & \\
\hline & \multirow[t]{3}{*}{ PT Eccentric KF } & $\mathrm{G}+24 \mathrm{H}$ & -0.83 & & & $1 / 1$ & Limited & 20 & & & \\
\hline & & $\mathrm{G}+48 \mathrm{H}$ & 0.60 & & & $1 / 1$ & Limited & 20 & & & \\
\hline & & $\mathrm{G}+72 \mathrm{H}$ & 0.58 & & & $1 / 1$ & Limited & 20 & & & \\
\hline \multirow[t]{11}{*}{ VHIR } & \multirow[t]{3}{*}{ Muscle damage } & Post & 0.67 & 0.40 & 0.94 & $5 / 3$ & Moderate & 45 & 77 & 8 & 0.02 \\
\hline & & $\mathrm{G}+24 \mathrm{H}$ & 0.54 & 0.35 & 0.65 & $5 / 6$ & Strong & 87 & 0 & 2.80 & 0.73 \\
\hline & & $\mathrm{G}+48 \mathrm{H}$ & 0.36 & 0.19 & 0.53 & $5 / 7$ & Moderate & 87 & 0 & 4.55 & 0.47 \\
\hline & \multirow[t]{2}{*}{$C M J_{P P O}$} & $\mathrm{G}+24 \mathrm{H}$ & -0.52 & -0.64 & -0.40 & $2 / 5$ & Strong & 67 & 0 & 2.21 & 0.70 \\
\hline & & $\mathrm{G}+48 \mathrm{H}$ & -0.25 & -0.48 & -0.02 & $2 / 5$ & Limited & 67 & 0 & 4.63 & 0.33 \\
\hline & \multirow[t]{3}{*}{ MVIC Dom } & $\mathrm{G}+24 \mathrm{H}$ & -0.57 & & & $1 / 1$ & Limited & 20 & & & \\
\hline & & $\mathrm{G}+48 \mathrm{H}$ & 0.45 & & & $1 / 1$ & Limited & 20 & & & \\
\hline & & $\mathrm{G}+72 \mathrm{H}$ & 0.43 & & & $1 / 1$ & Limited & 20 & & & \\
\hline & \multirow[t]{2}{*}{ Leucocytes count } & $\mathrm{G}+24 \mathrm{H}$ & -0.12 & & & $2 / 1$ & No & 39 & & & \\
\hline & & $\mathrm{G}+48 \mathrm{H}$ & 0.19 & & & $2 / 1$ & No & 39 & & & \\
\hline & BAM+ & $\mathrm{G}+24 \mathrm{H}$ & -0.21 & & & $1 / 1$ & No & 35 & & & \\
\hline
\end{tabular}


Table 4 Summary of findings from meta-analysis describing the relationship between external load metrics of level 1 [3] and fatigue-related markers (Continued)

\begin{tabular}{|c|c|c|c|c|c|c|c|c|c|}
\hline Predictors & Fatigue-related markers & Time & $\overline{\mathrm{r}}$ & Lower CL & Upper CL & Number of studies/correlations & Evidence & $\begin{array}{lll}n & P^{2} \quad \text { chi }^{2}\end{array}$ & $p$ \\
\hline & & $\mathrm{G}+48 \mathrm{H}$ & -0.17 & & & $1 / 1$ & No & 35 & \\
\hline & Cortisol & Post & NS & & & $2 / 0$ & No & & \\
\hline & & $\mathrm{G}+24 \mathrm{H}$ & NS & & & $1 / 0$ & No & & \\
\hline & & $\mathrm{G}+48 \mathrm{H}$ & NS & & & $1 / 0$ & No & & \\
\hline & Testosterone & Post & NS & & & $2 / 0$ & No & & \\
\hline & & $\mathrm{G}+24 \mathrm{H}$ & NS & & & $1 / 0$ & No & & \\
\hline & & $\mathrm{G}+48 \mathrm{H}$ & NS & & & $1 / 0$ & No & & \\
\hline & CMJ performance & Post & NS & & & $1 / 0$ & No & & \\
\hline & & $\mathrm{G}+24 \mathrm{H}$ & NS & & & $1 / 0$ & No & & \\
\hline & & $\mathrm{G}+48 \mathrm{H}$ & NS & & & $1 / 0$ & No & & \\
\hline & DOMS & $\mathrm{G}+24 \mathrm{H}$ & 0.07 & & & $1 / 1$ & No & 20 & \\
\hline & & $\mathrm{G}+48 \mathrm{H}$ & 0.07 & & & $1 / 1$ & No & 20 & \\
\hline & & $\mathrm{G}+72 \mathrm{H}$ & -0.10 & & & $1 / 1$ & No & 20 & \\
\hline & PT Concentric KE & $\mathrm{G}+24 \mathrm{H}$ & -0.69 & & & $1 / 1$ & Limited & 20 & \\
\hline & & $\mathrm{G}+48 \mathrm{H}$ & 0.51 & & & $1 / 1$ & Limited & 20 & \\
\hline & & $\mathrm{G}+72 \mathrm{H}$ & 0.51 & & & $1 / 1$ & Limited & 20 & \\
\hline & PT Concentric KF & $\mathrm{G}+24 \mathrm{H}$ & -0.78 & & & $1 / 1$ & Limited & 20 & \\
\hline & & $\mathrm{G}+48 \mathrm{H}$ & 0.72 & & & $1 / 1$ & Limited & 20 & \\
\hline & & $\mathrm{G}+72 \mathrm{H}$ & 0.61 & & & $1 / 1$ & Limited & 20 & \\
\hline & PT Eccentric KE & $\mathrm{G}+24 \mathrm{H}$ & -0.59 & & & $1 / 1$ & Limited & 20 & \\
\hline & & $\mathrm{G}+48 \mathrm{H}$ & 0.51 & & & $1 / 1$ & Limited & 20 & \\
\hline & & $\mathrm{G}+72 \mathrm{H}$ & 0.48 & & & $1 / 1$ & Limited & 20 & \\
\hline & PT Eccentric KF & $\mathrm{G}+24 \mathrm{H}$ & -0.83 & & & $1 / 1$ & Limited & 20 & \\
\hline & & $\mathrm{G}+48 \mathrm{H}$ & 0.63 & & & $1 / 1$ & Limited & 20 & \\
\hline & & $\mathrm{G}+72 \mathrm{H}$ & 0.62 & & & $1 / 1$ & Limited & 20 & \\
\hline VHIR dist/min & Muscle damage & $\mathrm{G}+24 \mathrm{H}$ & 0.37 & 0.11 & 0.55 & $2 / 5$ & Limited & 67 & \\
\hline & & $\mathrm{G}+48 \mathrm{H}$ & 0.13 & 0.05 & 0.31 & $2 / 5$ & Limited & 67 & \\
\hline & $C M J_{P P O}$ & $\mathrm{G}+24 \mathrm{H}$ & -0.22 & -0.48 & -0.03 & $2 / 5$ & Limited & 67 & \\
\hline & & $\mathrm{G}+48 \mathrm{H}$ & -0.25 & -0.53 & 0.05 & $2 / 5$ & Limited & 67 & \\
\hline & BAM+ & $\mathrm{G}+24 \mathrm{H}$ & -0.44 & & & $1 / 1$ & Limited & 35 & \\
\hline & & $\mathrm{G}+48 \mathrm{H}$ & -0.40 & & & $1 / 1$ & Limited & 35 & \\
\hline VHIR runs/min & Muscle damage & $\mathrm{G}+24 \mathrm{H}$ & 0.24 & 0.06 & 0.42 & $2 / 5$ & Limited & 67 & \\
\hline & & $\mathrm{G}+48 \mathrm{H}$ & 0.11 & -0.05 & 0.29 & $2 / 5$ & Limited & 67 & \\
\hline & CMJ peak power & $\mathrm{G}+24 \mathrm{H}$ & -0.22 & -0.49 & -0.05 & $2 / 5$ & Limited & 67 & \\
\hline & & $\mathrm{G}+48 \mathrm{H}$ & -0.20 & -0.41 & -0.02 & $2 / 5$ & Limited & 67 & \\
\hline & Cortisol & Post & NS & & & $1 / 1$ & No & & \\
\hline & Testosterone & Post & NS & & & $1 / 1$ & No & & \\
\hline & $\mathrm{BAM}+$ & $\mathrm{G}+24 \mathrm{H}$ & -0.55 & & & $1 / 1$ & Limited & 35 & \\
\hline & & $\mathrm{G}+48 \mathrm{H}$ & -0.49 & & & $1 / 1$ & Limited & 35 & \\
\hline Sprint & Muscle damage & Post & 0.12 & & & $2 / 1$ & Limited & 35 & \\
\hline & & $\mathrm{G}+24 \mathrm{H}$ & 0.40 & & & $2 / 1$ & Limited & 35 & \\
\hline & & $\mathrm{G}+48 \mathrm{H}$ & 0.38 & & & $3 / 2$ & Limited & 45 & \\
\hline & & $\mathrm{G}+72 \mathrm{H}$ & -0.02 & & & $1 / 1$ & Limited & 10 & \\
\hline
\end{tabular}


Table 4 Summary of findings from meta-analysis describing the relationship between external load metrics of level 1 [3] and fatigue-related markers (Continued)

\begin{tabular}{|c|c|c|c|c|c|c|c|}
\hline Predictors & Fatigue-related markers & Time & $\overline{\mathrm{r}}$ & Lower CL Upper CL & Number of studies/correlations & Evidence & $\begin{array}{lll}n & R^{2} \quad \mathrm{chi}^{2}\end{array}$ \\
\hline & \multirow[t]{3}{*}{ MVIC } & $G+24 H$ & -0.51 & & $2 / 1$ & Limited & 20 \\
\hline & & $\mathrm{G}+48 \mathrm{H}$ & 0.35 & & $2 / 1$ & Limited & 20 \\
\hline & & $\mathrm{G}+72 \mathrm{H}$ & 0.35 & & $2 / 1$ & Limited & 20 \\
\hline & \multirow[t]{3}{*}{ Leucocyte count } & $G+24 H$ & -0.10 & & $1 / 1$ & Limited & 20 \\
\hline & & $\mathrm{G}+48 \mathrm{H}$ & 0.17 & & $1 / 1$ & Limited & 20 \\
\hline & & $\mathrm{G}+72 \mathrm{H}$ & -0.23 & & $1 / 1$ & Limited & 20 \\
\hline & \multirow[t]{3}{*}{ DOMS } & $\mathrm{G}+24 \mathrm{H}$ & 0.31 & & $2 / 2$ & Limited & 20 \\
\hline & & $\mathrm{G}+48 \mathrm{H}$ & 0.05 & & $2 / 1$ & Limited & 20 \\
\hline & & $\mathrm{G}+72 \mathrm{H}$ & -0.37 & & $2 / 1$ & Limited & 20 \\
\hline & \multirow[t]{3}{*}{ PT Concentric KE } & $\mathrm{G}+24 \mathrm{H}$ & -0.58 & & $1 / 1$ & Limited & 20 \\
\hline & & $\mathrm{G}+48 \mathrm{H}$ & 0.55 & & $1 / 1$ & Limited & 20 \\
\hline & & $\mathrm{G}+72 \mathrm{H}$ & 0.58 & & $1 / 1$ & Limited & 20 \\
\hline & \multirow[t]{3}{*}{ PT Concentric KF } & $G+24 H$ & -0.70 & & $1 / 1$ & Limited & 20 \\
\hline & & $\mathrm{G}+48 \mathrm{H}$ & 0.70 & & $1 / 1$ & Limited & 20 \\
\hline & & $\mathrm{G}+72 \mathrm{H}$ & 0.56 & & $1 / 1$ & Limited & 20 \\
\hline & \multirow[t]{3}{*}{ PT Eccentric KE } & $\mathrm{G}+24 \mathrm{H}$ & -0.45 & & $1 / 1$ & Limited & 20 \\
\hline & & $\mathrm{G}+48 \mathrm{H}$ & 0.64 & & $1 / 1$ & Limited & 20 \\
\hline & & $\mathrm{G}+72 \mathrm{H}$ & 0.45 & & $1 / 1$ & Limited & 20 \\
\hline & \multirow[t]{3}{*}{ PT Eccentric KF } & $G+24 H$ & -0.68 & & $1 / 1$ & Limited & 20 \\
\hline & & $\mathrm{G}+48 \mathrm{H}$ & 0.63 & & $1 / 1$ & Limited & 20 \\
\hline & & $\mathrm{G}+72 \mathrm{H}$ & 0.61 & & $1 / 1$ & Limited & 20 \\
\hline & \multirow[t]{4}{*}{ CMJ performance } & Post & NS & & $2 / 0$ & No & \\
\hline & & $G+24 H$ & NS & & $2 / 0$ & No & \\
\hline & & $\mathrm{G}+48 \mathrm{H}$ & 0.27 & & $3 / 1$ & No & 10 \\
\hline & & $\mathrm{G}+72 \mathrm{H}$ & 0.27 & & $3 / 1$ & No & 10 \\
\hline & \multirow[t]{3}{*}{ TQR } & $G+24 H$ & NS & & $1 / 0$ & No & \\
\hline & & $\mathrm{G}+48 \mathrm{H}$ & NS & & $1 / 0$ & No & \\
\hline & & $\mathrm{G}+72 \mathrm{H}$ & NS & & $1 / 0$ & No & \\
\hline
\end{tabular}

$\overline{\bar{r}}$. pooled correlation from Hunter and Schmidt random-effects method, CL: confidence limit, $N$ : number of players, TD: total distance, KE: knee extension, KF: knee flexion, CMJ: countermovement jump, $C \mathrm{JJ}_{\mathrm{Ppo}}$ : countermovement jump peak power, PT: peak torque, DOMS: delayed onset muscle soreness, BAM+: brief assessment of the mood, HIR: high-intensity running $\left(>4 \mathrm{~m} \mathrm{~s}^{-1}\right)$, MVIC: maximal voluntary isometric contraction, VHIR: very high-intensity running $\left(>5.5 \mathrm{~m} \mathrm{~s}{ }^{-1}\right)$, conc: concentric, ecc: eccentric, Acc: acceleration, Decel: deceleration, No: no evidence, NS: not specified, PT 1-100 Hz: peak torque induced by single (1 Hz) and paired electrical stimuli $(10$ and $100 \mathrm{~Hz})$

\section{Total Distance}

In relation to absolute $\mathrm{TD}$, muscle damage markers and $\mathrm{CMJ}_{\mathrm{PPO}}$ were assessed in at least two studies ( $n$ = 159 match observations, Table 4). Pooled data showed limited evidence of small (i.e., $\mathrm{G}+48 \mathrm{H}$ ) to moderate (Post and $\mathrm{G}+24 \mathrm{H}$ ) correlations between TD and post-match muscle damage markers (Table 4). The relationships between $\mathrm{TD}$ and $\mathrm{CMJ}_{\mathrm{PPO}}$ were rated as trivial (i.e., $\mathrm{G}+24 \mathrm{H}$ ) to small (i.e., $\mathrm{G}+$ $48 \mathrm{H}$ ). The correlation between relative TD (i.e., TD/ min) and $\mathrm{CMJ}_{\mathrm{PPO}}$ was rated as trivial, similarly to the correlation between relative TD and muscle damage markers (Table 4).

\section{High-Intensity Running ( $>4 \mathrm{~m} \mathrm{~s}^{-1}$ ):}

In relation to HIR, muscle damage markers, DOMS and MVIC were assessed in at least two studies ( $n=93$ match observations). Pooled data showed strong evidence of a large correlation between HIR and muscle damage at Post $(\bar{r}=0.59 ; 95 \% \mathrm{CI}=[0.35,0.84])$. At $\mathrm{G}+$ $24 \mathrm{H}$ and $\mathrm{G}+48 \mathrm{H}$, the magnitude of this relationship became moderate with limited evidence (Table 4). Between HIR and MVIC, there was limited evidence of a negative and large correlation at $\mathrm{G}+24 \mathrm{H}$ and positively moderate correlations at $\mathrm{G}+48 \mathrm{H}$ and $\mathrm{G}+72 \mathrm{H}$. The relationship between HIR and DOMS was rated as trivial at all time points (Table 4). 
Table 5 Summary of findings from meta-analysis describing the relationship between external load metrics (levels 2 and 3) [3] and fatigue-related markers

\begin{tabular}{|c|c|c|c|c|c|c|c|c|c|c|c|}
\hline Predictors & Fatigue-related markers & Time & $\overline{\mathrm{r}}$ & Lower CL & Upper CL & Number of studies/correlations & Evidence & $n$ & $P^{2}$ & $\mathrm{chi}^{2}$ & $p$ \\
\hline \multirow[t]{33}{*}{$\mathrm{HI}$ ACC } & \multirow[t]{4}{*}{ Muscle damage } & Post & 0.24 & 0.02 & 0.46 & $2 / 1$ & Limited & 45 & & & \\
\hline & & $\mathrm{G}+24 \mathrm{H}$ & 0.41 & 0.30 & 0.51 & $3 / 5$ & Moderate & 52 & 0 & 0.97 & 0.91 \\
\hline & & $\mathrm{G}+48 \mathrm{H}$ & 0.30 & 0.15 & 0.45 & $3 / 6$ & Moderate & 77 & 0 & 2.95 & 0.71 \\
\hline & & $\mathrm{G}+72 \mathrm{H}$ & 0.01 & & & $1 / 1$ & Limited & 10 & & & \\
\hline & \multirow[t]{2}{*}{$C M J_{P P O}$} & $\mathrm{G}+24 \mathrm{H}$ & -0.61 & -0.79 & -0.42 & $2 / 5$ & Strong & 46 & 24 & 4 & 0.41 \\
\hline & & $\mathrm{G}+48 \mathrm{H}$ & -0.36 & -0.68 & -0.05 & $2 / 4$ & Limited & 32 & 0 & 3.71 & 0.29 \\
\hline & \multirow[t]{3}{*}{$M V I C$} & $\mathrm{G}+24 \mathrm{H}$ & -0.79 & & & $2 / 1$ & Limited & 34 & & & \\
\hline & & $\mathrm{G}+48 \mathrm{H}$ & 0.74 & & & $2 / 1$ & Limited & 34 & & & \\
\hline & & $\mathrm{G}+72 \mathrm{H}$ & 0.46 & & & $2 / 1$ & Limited & 34 & & & \\
\hline & \multirow[t]{3}{*}{ DOMS } & $\mathrm{G}+24 \mathrm{H}$ & -0.05 & & & $3 / 1$ & Limited & 20 & & & \\
\hline & & $\mathrm{G}+48 \mathrm{H}$ & 0.23 & -0.27 & 0.73 & $3 / 2$ & Limited & 34 & 79 & 4.04 & 0.04 \\
\hline & & $\mathrm{G}+72 \mathrm{H}$ & 0.23 & -0.11 & 0.56 & $3 / 2$ & Limited & 34 & 43 & 1.84 & 0.17 \\
\hline & \multirow[t]{3}{*}{ CMJ performance } & $\mathrm{G}+24 \mathrm{H}$ & NS & & & $2 / 0$ & No & & & & \\
\hline & & $\mathrm{G}+48 \mathrm{H}$ & 0.27 & & & $3 / 1$ & Limited & 10 & & & \\
\hline & & $\mathrm{G}+72 \mathrm{H}$ & 0.09 & & & $1 / 1$ & Limited & 10 & & & \\
\hline & \multirow[t]{3}{*}{ Leucocytes count } & $\mathrm{G}+24 \mathrm{H}$ & 0.17 & & & $1 / 1$ & Limited & 20 & & & \\
\hline & & $\mathrm{G}+48 \mathrm{H}$ & -0.14 & & & $1 / 1$ & Limited & 20 & & & \\
\hline & & $\mathrm{G}+72 \mathrm{H}$ & -0.02 & & & $1 / 1$ & Limited & 20 & & & \\
\hline & \multirow[t]{3}{*}{ PT Concentric KE } & $\mathrm{G}+24 \mathrm{H}$ & -0.75 & & & $1 / 1$ & Limited & 20 & & & \\
\hline & & $\mathrm{G}+48 \mathrm{H}$ & 0.52 & & & $1 / 1$ & Limited & 20 & & & \\
\hline & & $\mathrm{G}+72 \mathrm{H}$ & 0.56 & & & $1 / 1$ & Limited & 20 & & & \\
\hline & \multirow[t]{3}{*}{ PT Concentric KF } & $\mathrm{G}+24 \mathrm{H}$ & -0.84 & & & $1 / 1$ & Limited & 20 & & & \\
\hline & & $\mathrm{G}+48 \mathrm{H}$ & 0.70 & & & $1 / 1$ & Limited & 20 & & & \\
\hline & & $\mathrm{G}+72 \mathrm{H}$ & 0.58 & & & $1 / 1$ & Limited & 20 & & & \\
\hline & \multirow[t]{3}{*}{ PT Eccentric KE } & $\mathrm{G}+24 \mathrm{H}$ & -0.64 & & & $1 / 1$ & Limited & 20 & & & \\
\hline & & $\mathrm{G}+48 \mathrm{H}$ & 0.51 & & & $1 / 1$ & Limited & 20 & & & \\
\hline & & $\mathrm{G}+72 \mathrm{H}$ & 0.41 & & & $1 / 1$ & Limited & 20 & & & \\
\hline & \multirow[t]{3}{*}{ PT Eccentric KF } & $\mathrm{G}+24 \mathrm{H}$ & -0.89 & & & $1 / 1$ & Limited & 20 & & & \\
\hline & & $\mathrm{G}+48 \mathrm{H}$ & 0.62 & & & $1 / 1$ & Limited & 20 & & & \\
\hline & & $\mathrm{G}+72 \mathrm{H}$ & 0.69 & & & $1 / 1$ & Limited & 20 & & & \\
\hline & \multirow[t]{3}{*}{ TQR } & $\mathrm{G}+24 \mathrm{H}$ & NS & & & $1 / 0$ & No & & & & \\
\hline & & $\mathrm{G}+48 \mathrm{H}$ & NS & & & $1 / 0$ & No & & & & \\
\hline & & $\mathrm{G}+72 \mathrm{H}$ & NS & & & $1 / 0$ & No & & & & \\
\hline \multirow[t]{10}{*}{ Mod to HI Acc } & \multirow[t]{3}{*}{ Muscle damage } & Post & 0.34 & & & $2 / 1$ & Limited & 35 & & & \\
\hline & & $\mathrm{G}+24 \mathrm{H}$ & 0.32 & & & $2 / 1$ & Limited & 35 & & & \\
\hline & & $\mathrm{G}+48 \mathrm{H}$ & 0.27 & & & $2 / 1$ & Limited & 35 & & & \\
\hline & CMJ conc force & $\mathrm{G}+48 \mathrm{H}$ & -0.49 & -0.78 & -0.17 & $1 / 1$ & Limited & 35 & & & \\
\hline & CMJ ecc force & Post & -0.47 & -0.73 & -0.11 & $1 / 1$ & Limited & 35 & & & \\
\hline & \multirow[t]{3}{*}{$M V I C$} & $\mathrm{G}+24 \mathrm{H}$ & -0.74 & & & $1 / 1$ & Limited & 35 & & & \\
\hline & & $\mathrm{G}+48 \mathrm{H}$ & 0.80 & & & $1 / 1$ & Limited & 35 & & & \\
\hline & & $\mathrm{G}+72 \mathrm{H}$ & 0.49 & & & $1 / 1$ & Limited & 35 & & & \\
\hline & \multirow[t]{2}{*}{ DOMS } & $\mathrm{G}+24 \mathrm{H}$ & -0.04 & & & $1 / 1$ & Limited & 35 & & & \\
\hline & & $\mathrm{G}+48 \mathrm{H}$ & -0.02 & & & $1 / 1$ & Limited & 35 & & & \\
\hline
\end{tabular}


Table 5 Summary of findings from meta-analysis describing the relationship between external load metrics (levels 2 and 3) [3] and fatigue-related markers (Continued)

\begin{tabular}{|c|c|c|c|c|c|c|c|c|c|c|c|}
\hline Predictors & Fatigue-related markers & Time & $\overline{\mathrm{r}}$ & Lower CL & Upper CL & Number of studies/correlations & Evidence & $n$ & $P^{2}$ & $\mathrm{chi}^{2}$ & $p$ \\
\hline & & $\mathrm{G}+72 \mathrm{H}$ & -0.04 & & & $1 / 1$ & Limited & 35 & & & \\
\hline & PT Concentric KE & $\mathrm{G}+24 \mathrm{H}$ & -0.75 & & & $1 / 1$ & Limited & 20 & & & \\
\hline & & $\mathrm{G}+48 \mathrm{H}$ & 0.56 & & & $1 / 1$ & Limited & 20 & & & \\
\hline & & $\mathrm{G}+72 \mathrm{H}$ & 0.64 & & & $1 / 1$ & Limited & 20 & & & \\
\hline & PT Concentric KF & $\mathrm{G}+24 \mathrm{H}$ & -0.84 & & & $1 / 1$ & Limited & 20 & & & \\
\hline & & $\mathrm{G}+48 \mathrm{H}$ & 0.70 & & & $1 / 1$ & Limited & 20 & & & \\
\hline & & $\mathrm{G}+72 \mathrm{H}$ & 0.57 & & & $1 / 1$ & Limited & 20 & & & \\
\hline & PT Eccentric KE & $\mathrm{G}+24 \mathrm{H}$ & -0.60 & & & $1 / 1$ & Limited & 20 & & & \\
\hline & & $\mathrm{G}+48 \mathrm{H}$ & 0.57 & & & $1 / 1$ & Limited & 20 & & & \\
\hline & & $\mathrm{G}+72 \mathrm{H}$ & 0.47 & & & $1 / 1$ & Limited & 20 & & & \\
\hline & PT Eccentric KF & $G+24 H$ & -0.85 & & & $1 / 1$ & Limited & 20 & & & \\
\hline & & $\mathrm{G}+48 \mathrm{H}$ & 0.65 & & & $1 / 1$ & Limited & 20 & & & \\
\hline & & $\mathrm{G}+72 \mathrm{H}$ & 0.68 & & & $1 / 1$ & Limited & 20 & & & \\
\hline \multirow[t]{30}{*}{ HI Decel } & Muscle damage & Post & 0.32 & 0.13 & 0.51 & $3 / 2$ & Limited & 45 & & & \\
\hline & & $\mathrm{G}+24 \mathrm{H}$ & 0.47 & 0.36 & 0.59 & $5 / 6$ & Strong & 52 & 0 & 1.26 & 0.87 \\
\hline & & $\mathrm{G}+48 \mathrm{H}$ & 0.37 & 0.18 & 0.55 & $6 / 7$ & Moderate & 67 & 0 & 4.8 & 0.44 \\
\hline & & $\mathrm{G}+72 \mathrm{H}$ & 0.00 & & & $1 / 1$ & Limited & 10 & & & \\
\hline & $C M J_{P P O}$ & $\mathrm{G}+24 \mathrm{H}$ & -0.59 & -0.78 & 0.40 & $1 / 4$ & Limited & 32 & 0 & 2.49 & 0.48 \\
\hline & & $\mathrm{G}+48 \mathrm{H}$ & -0.11 & -0.42 & 0.19 & $1 / 4$ & Limited & 32 & 0 & 2.63 & 0.45 \\
\hline & CMJ performance & $\mathrm{G}+24 \mathrm{H}$ & NS & & & $1 / 0$ & No & & & & \\
\hline & & $\mathrm{G}+48 \mathrm{H}$ & 0.15 & & & $2 / 1$ & Limited & 10 & & & \\
\hline & & $\mathrm{G}+72 \mathrm{H}$ & 0.16 & & & $1 / 1$ & Limited & 10 & & & \\
\hline & MVIC & $\mathrm{G}+24 \mathrm{H}$ & -0.78 & & & $2 / 1$ & Limited & 34 & & & \\
\hline & & $\mathrm{G}+48 \mathrm{H}$ & 0.70 & & & $2 / 1$ & Limited & 34 & & & \\
\hline & & $\mathrm{G}+72 \mathrm{H}$ & 0.46 & & & $2 / 1$ & Limited & 34 & & & \\
\hline & DOMS & $\mathrm{G}+24 \mathrm{H}$ & -0.11 & & & $2 / 1$ & Limited & 34 & & & \\
\hline & & $\mathrm{G}+48 \mathrm{H}$ & 0.08 & & & $2 / 1$ & Limited & 34 & & & \\
\hline & PT Concentric KE & $\mathrm{G}+24 \mathrm{H}$ & -0.82 & & & $1 / 1$ & Limited & 20 & & & \\
\hline & & $\mathrm{G}+48 \mathrm{H}$ & 0.43 & & & $1 / 1$ & Limited & 20 & & & \\
\hline & & $\mathrm{G}+72 \mathrm{H}$ & 0.59 & & & $1 / 1$ & Limited & 20 & & & \\
\hline & PT Concentric KF & $G+24 H$ & -0.84 & & & $1 / 1$ & Limited & 20 & & & \\
\hline & & $\mathrm{G}+48 \mathrm{H}$ & 0.70 & & & $1 / 1$ & Limited & 20 & & & \\
\hline & & $\mathrm{G}+72 \mathrm{H}$ & 0.58 & & & $1 / 1$ & Limited & 20 & & & \\
\hline & PT Eccentric KE & $\mathrm{G}+24 \mathrm{H}$ & -0.55 & & & $1 / 1$ & Limited & 20 & & & \\
\hline & & $\mathrm{G}+48 \mathrm{H}$ & 0.53 & & & $1 / 1$ & Limited & 20 & & & \\
\hline & & $\mathrm{G}+72 \mathrm{H}$ & 0.53 & & & $1 / 1$ & Limited & 20 & & & \\
\hline & PT Eccentric KF & $\mathrm{G}+24 \mathrm{H}$ & -0.82 & & & $1 / 1$ & Limited & 20 & & & \\
\hline & & $\mathrm{G}+48 \mathrm{H}$ & 0.62 & & & $1 / 1$ & Limited & 20 & & & \\
\hline & & $\mathrm{G}+72 \mathrm{H}$ & 0.65 & & & $1 / 1$ & Limited & 20 & & & \\
\hline & Leucocytes count & $\mathrm{G}+24 \mathrm{H}$ & 0.22 & & & $1 / 1$ & Limited & 20 & & & \\
\hline & & $\mathrm{G}+48 \mathrm{H}$ & 0.14 & & & $1 / 1$ & Limited & 20 & & & \\
\hline & & $\mathrm{G}+72 \mathrm{H}$ & -0.04 & & & $1 / 1$ & Limited & 20 & & & \\
\hline & TQR & $G+24 H$ & NS & & & $1 / 0$ & No & & & & \\
\hline
\end{tabular}


Table 5 Summary of findings from meta-analysis describing the relationship between external load metrics (levels 2 and 3) [3] and fatigue-related markers (Continued)

\begin{tabular}{|c|c|c|c|c|c|c|c|c|c|c|c|}
\hline Predictors & Fatigue-related markers & Time & $\overline{\mathrm{r}}$ & Lower CL & Upper CL & Number of studies/correlations & Evidence & $n$ & $P^{2}$ & $\mathrm{chi}^{2}$ & $p$ \\
\hline & & $\mathrm{G}+48 \mathrm{H}$ & NS & & & $1 / 0$ & No & & & & \\
\hline & & $\mathrm{G}+72 \mathrm{H}$ & NS & & & $1 / 0$ & No & & & & \\
\hline \multirow[t]{23}{*}{ Mod to HI Decel } & Muscle damage & Post & 0.14 & & & $2 / 1$ & Limited & 35 & & & \\
\hline & & $\mathrm{G}+24 \mathrm{H}$ & 0.03 & & & $2 / 1$ & Limited & 35 & & & \\
\hline & & $\mathrm{G}+48 \mathrm{H}$ & 0.25 & & & $2 / 2$ & Limited & 67 & & & \\
\hline & MVIC & $\mathrm{G}+24 \mathrm{H}$ & -0.80 & & & $1 / 1$ & Limited & 35 & & & \\
\hline & & $\mathrm{G}+48 \mathrm{H}$ & 0.76 & & & $1 / 1$ & Limited & 35 & & & \\
\hline & & $\mathrm{G}+72 \mathrm{H}$ & 0.39 & & & $1 / 1$ & Limited & 35 & & & \\
\hline & CMJ conc force & $\mathrm{G}+48 \mathrm{H}$ & -0.49 & -0.78 & -0.17 & $1 / 1$ & Limited & 35 & & & \\
\hline & CMJ ecc force & Post & -0.47 & -0.73 & -0.11 & $1 / 1$ & Limited & 35 & & & \\
\hline & DOMS & $\mathrm{G}+24 \mathrm{H}$ & -0.14 & & & $1 / 1$ & Limited & 35 & & & \\
\hline & & $\mathrm{G}+48 \mathrm{H}$ & 0.06 & & & $1 / 1$ & Limited & 35 & & & \\
\hline & & $\mathrm{G}+72 \mathrm{H}$ & 0.10 & & & $1 / 1$ & Limited & 35 & & & \\
\hline & PT Concentric KE & $\mathrm{G}+24 \mathrm{H}$ & -0.82 & & & $1 / 1$ & Limited & 20 & & & \\
\hline & & $\mathrm{G}+48 \mathrm{H}$ & 0.40 & & & $1 / 1$ & Limited & 20 & & & \\
\hline & & $\mathrm{G}+72 \mathrm{H}$ & 0.59 & & & $1 / 1$ & Limited & 20 & & & \\
\hline & PT Concentric KF & $\mathrm{G}+24 \mathrm{H}$ & -0.83 & & & $1 / 1$ & Limited & 20 & & & \\
\hline & & $\mathrm{G}+48 \mathrm{H}$ & 0.64 & & & $1 / 1$ & Limited & 20 & & & \\
\hline & & $\mathrm{G}+72 \mathrm{H}$ & 0.57 & & & $1 / 1$ & Limited & 20 & & & \\
\hline & PT Eccentric KE & $\mathrm{G}+24 \mathrm{H}$ & -0.54 & & & $1 / 1$ & Limited & 20 & & & \\
\hline & & $\mathrm{G}+48 \mathrm{H}$ & 0.53 & & & $1 / 1$ & Limited & 20 & & & \\
\hline & & $\mathrm{G}+72 \mathrm{H}$ & 0.42 & & & $1 / 1$ & Limited & 20 & & & \\
\hline & PT Eccentric KF & $G+24 H$ & -0.84 & & & $1 / 1$ & Limited & 20 & & & \\
\hline & & $\mathrm{G}+48 \mathrm{H}$ & 0.57 & & & $1 / 1$ & Limited & 20 & & & \\
\hline & & $\mathrm{G}+72 \mathrm{H}$ & 0.71 & & & $1 / 1$ & Limited & 20 & & & \\
\hline \multirow[t]{4}{*}{ High Impact } & Muscle damage & $\mathrm{G}+24 \mathrm{H}$ & 0.19 & -0.14 & 0.51 & $2 / 4$ & Limited & 47 & 0 & 3.17 & 0.37 \\
\hline & & $\mathrm{G}+48 \mathrm{H}$ & 0.15 & -0.22 & 0.53 & $2 / 4$ & Limited & 47 & 0 & 4.17 & 0.24 \\
\hline & $C M J_{P P O}$ & $\mathrm{G}+24 \mathrm{H}$ & -0.32 & -0.61 & -0.03 & $2 / 4$ & Limited & 50 & 5 & 5.60 & 0.23 \\
\hline & & $\mathrm{G}+48 \mathrm{H}$ & 0.04 & -0.30 & 0.38 & $1 / 4$ & Limited & 32 & 0 & 3.25 & 0.36 \\
\hline
\end{tabular}

$\bar{r}$. pooled correlation from Hunter and Schmidt random-effects method, $C L$ : confidence limit, $N$ : number of players, TD: total distance, KE: knee extension, KF: knee flexion, $C M J$ : countermovement jump, $C M J_{P P O}$ : countermovement jump peak power, $P T$ : peak torque, DOMS: delayed onset muscle soreness, $T Q R$ : total quality of recovery, BAM+: brief assessment of the mood, HI: high intensity, Mod: moderate, MVIC: maximal voluntary isometric contraction, conc: concentric, ecc: eccentric,

Acc: acceleration, Decel: deceleration, No: no evidence, Conf: conflicted

\section{Very High-Intensity Running $\left(>5.5 \mathrm{~m} \mathrm{~s}^{-1}\right)$ :}

In relation to absolute VHIR, muscle damage markers and $\mathrm{CMJ}_{\mathrm{PPO}}$ were assessed in at least two studies. Pooled data ( $n=146$ match observations) showed moderate to strong evidence of large correlations between VHIR and muscle damage at Post $(\bar{r}=0.67 ; 95 \% \mathrm{CI}=[0.40,0.94])$ and at $\mathrm{G}+24 \mathrm{H}(\bar{r}=0.54 ; 95 \% \mathrm{CI}=[0.35,0.65])$. At $\mathrm{G}+$ $48 \mathrm{H}$, the magnitude of this relationship became moderate with limited evidence. Between VHIR and $\mathrm{CMJ}_{\mathrm{PPO}}$, there was strong evidence of a negative and large pooled correlation $(=-0.52 ; 95 \% \mathrm{CI}=[-0.64,-0.40])$ at $\mathrm{G}$ $+24 \mathrm{H}$. This relationship was rated as moderate at $\mathrm{G}+$ $48 \mathrm{H}$ with conflicted evidence (Table 4).
The correlations between relative VHIR (per unit of time) and muscle damage markers and $\mathrm{CMJ}_{\mathrm{PPO}}$ output were rated as small, with limited evidence. Relative VHIR was moderately $(G+24 \mathrm{H})$ to largely $(G+48 \mathrm{H})$ correlated with the brief assessment of $\operatorname{mood}(1$ study, $n$ = 35), with limited evidence (Table 4).

\section{Sprint Running}

Muscle damage markers and MVIC were assessed with sprint running performance during the match. Data showed moderate to large correlations between sprint running distance and these two fatigue-related markers 


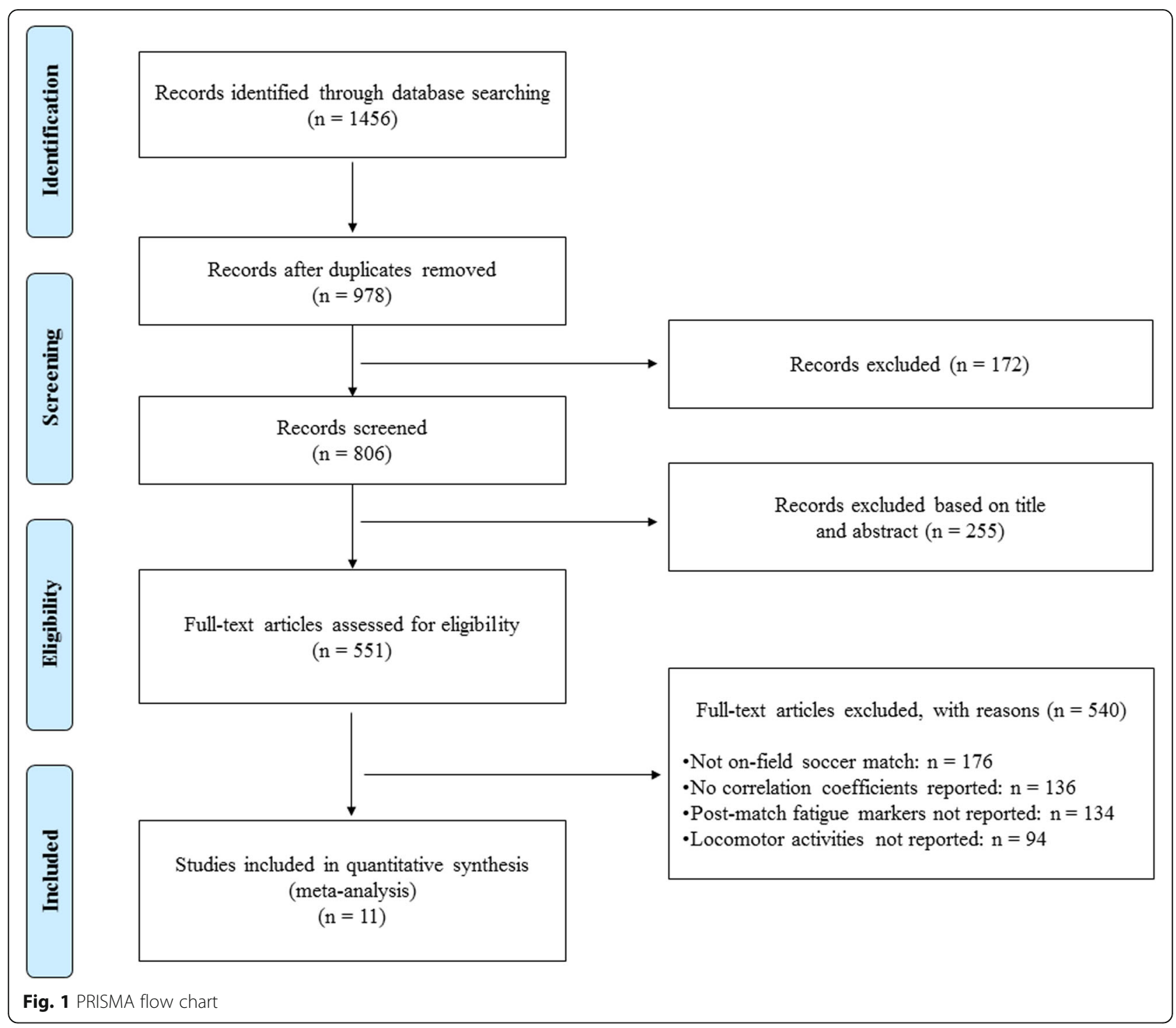

from $\mathrm{G}+24 \mathrm{H}$ to $\mathrm{G}+72 \mathrm{H}$, with limited evidence (Table $4)$.

\section{Acceleration Variables}

Acceleration variables were related to muscle damage markers, $\mathrm{CMJ}_{\mathrm{PPO}}, \mathrm{MVIC}$, and DOMS. Pooled data showed strong (i.e., at $\mathrm{G}+24 \mathrm{H}$ ) and limited (at Post and $\mathrm{G}+48 \mathrm{H}$ ) evidence of moderate correlations between high accelerations and muscle damage markers (Table 5).

There was moderate evidence of a large and negative correlation $(\bar{r}=-0.61 ; 95 \% \mathrm{CI}=[-0.82,-0.42])$ at $\mathrm{G}+$ $24 \mathrm{H}$ between $\mathrm{CMJ}_{\mathrm{PPO}}$ and high accelerations (Table 5). At $\mathrm{G}+48 \mathrm{H}$, this relationship was rated as moderate with limited evidence. There was limited evidence of moderate (i.e., $\mathrm{G}+72 \mathrm{H})$ to very large $(\mathrm{G}+24 \mathrm{H}$ and $\mathrm{G}+$ $48 \mathrm{H})$ correlations between high accelerations and MVIC of the dominant leg (Table 5). DOMS presented trivial to small correlations with high accelerations regardless of the time points.

\section{Deceleration Variables}

Deceleration variables were related to muscle damage markers, $\mathrm{CMJ}_{\mathrm{PPO}}$ and DOMS. Pooled data (3 studies, $n$ = 70 match observations) showed limited (at Post and G $+48 \mathrm{H}$ ) and moderate (i.e., at $\mathrm{G}+24 \mathrm{H}$ ) evidence of positive and moderate correlations between high decelerations and muscle damage markers. There was limited evidence of small $(\mathrm{G}+48 \mathrm{H})$ to large $(\mathrm{G}+24 \mathrm{H})$ correlations between high decelerations and $\mathrm{CMJ}_{\mathrm{PPO}}$. DOMS presented trivial correlations with high decelerations regardless of the time points (Table 5).

\section{High Impacts}

In relation to the number of high impacts, muscle damage markers and $\mathrm{CMJ}_{\mathrm{PPO}}$ were assessed. Pooled data (2 
studies, $n=40$ match observations) showed conflicted evidence of small correlations between high impact and muscle damage markers at $\mathrm{G}+24 \mathrm{H}$ and $\mathrm{G}+48 \mathrm{H}$. There was also conflicted evidence for the moderate correlation between high impacts and $\mathrm{CMJ}_{\mathrm{PPO}}$ at $\mathrm{G}+24 \mathrm{H}$ (Table 5).

\section{Regression Analysis}

The relationship between VHIR and fatigue-related markers (percentage change in $\mathrm{CMJ}_{\mathrm{PPO}}$ and $\mathrm{CK}$, Fig. 2) could be represented by a linear function (3 studies, 2 of high quality, 11 matches, $n=87$ player-match observations) (Fig. 2).

\section{Discussion}

The aim of this systematic review with meta-analysis was to examine whether external load metrics during soccer match-play reflect acute and residual changes in post-match biochemical, neuromuscular, and perceptual responses. The main findings were as follows: (1) the match-related running distance above $5.5 \mathrm{~m} \mathrm{~s}^{-1}$ was identified as the only monitoring variable largely

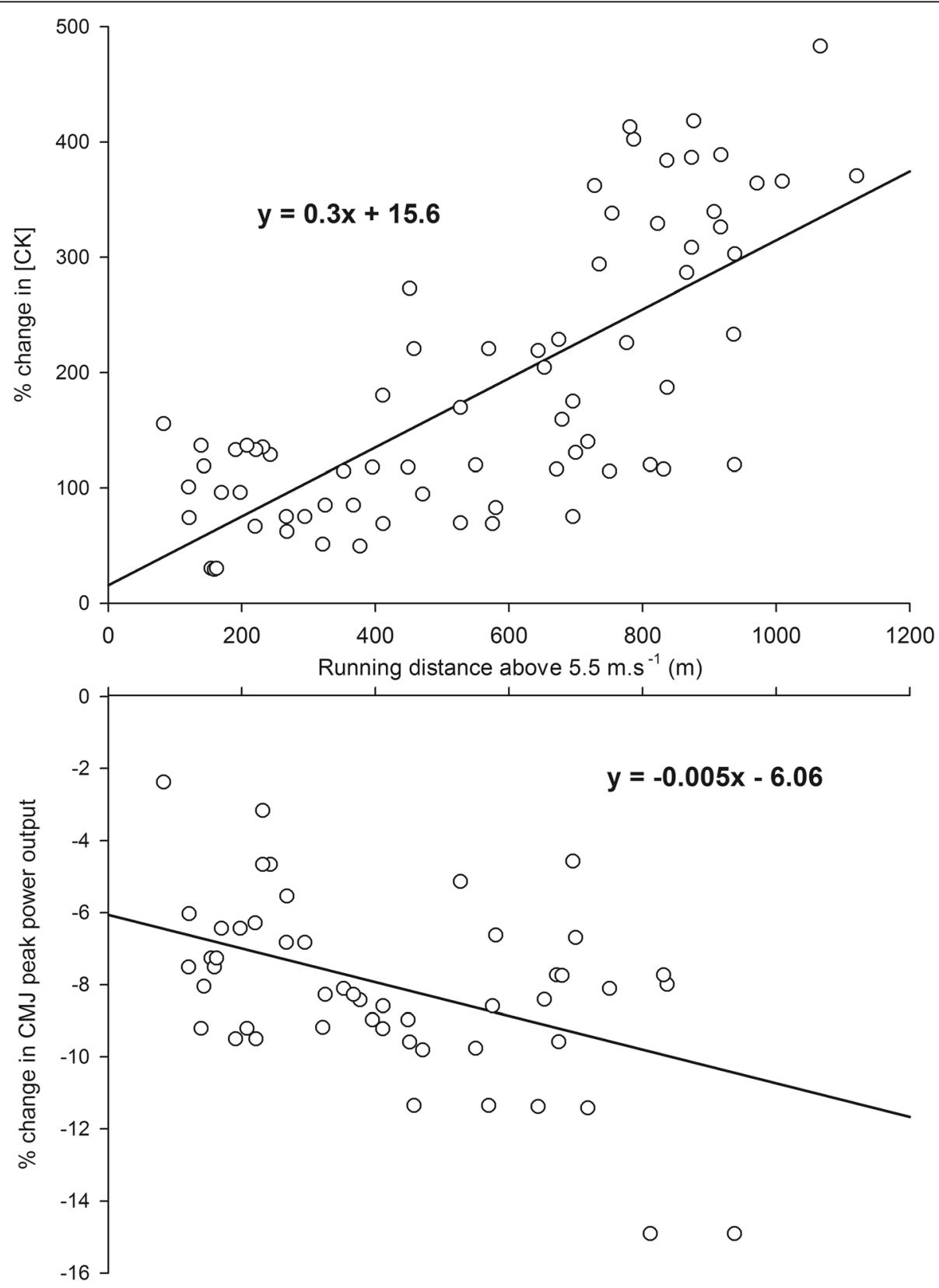

Fig. 2 Relationship between match-related running distance above $5.5 \mathrm{~m} \mathrm{~s}^{-1}$ and post-match changes in CK (upper panel) and CMJ peak power output (lower panel). CK: creatine kinase; CMJ: countermovement jump 
correlated with both biochemical and neuromuscular markers, (2) practically, at $\mathrm{G}+24 \mathrm{H}$, for each $100 \mathrm{~m}$ of VHI running distance covered, CK activity would increase by $30 \%$ and $\mathrm{CMJ}_{\mathrm{PPO}}$ would decrease by $0.5 \%$.

Among the biochemical variables systematically reviewed, there was strong evidence of two large pooled correlations ( $r=0.54$ to 0.67 ) between HIR or VHIR and changes in muscle damage markers (e.g., CK) at Post and $\mathrm{G}+24 \mathrm{H}$, respectively. These changes were also moderately correlated with high accelerations at G + $24 \mathrm{H}$. The weaker correlation may be due to the restricted number of selected studies examining acceleration variables. Over fifty listed variables, high accelerations (rank four) and VHIR (rank two) were reported as the monitored variables that were the most used by elite soccer team practitioners to quantify training and match loads [8]. From an injury prevention standpoint, hamstring strain injuries predominantly occur during VHIR and high acceleration [20, 42, 43]. Additionally, injury to the hamstrings muscle group is the most commonly reported injury in male soccer players $[42,44,45]$. Therefore, in the selected studies, the MVIC evaluation was focused on the hamstrings muscles (i.e., at $\mathrm{G}+24 \mathrm{H}$ and $\mathrm{G}+48 \mathrm{H}$ ) [21, 25]. Indeed, the injury mechanism may be due to the repeated and excessive lengthening demands placed on the hamstrings during the high eccentric force contractions involved during these specific efforts (e.g., VHIR) [43, 46]. Furthermore, as also supported by our results (Tables 4 and 5), exercise-induced muscle damage is more typically associated with the performance of fast eccentric muscle actions (e.g., high accelerations) than to the execution of lower velocity-based eccentric muscle actions (e.g., moderate accelerations) [47]. These fast eccentric muscle actions would exacerbate the mechanical stress characterized by cellular and subcellular structural disturbances, such as the focal disruption of the myofibers and cytoskeleton resulting in z-disk streaming [48, 49]. From our results, VHIR may represent the most sensitive external load metric to monitor changes in acute (immediately post-match) and residual (i.e., at $\mathrm{G}+24 \mathrm{H}$ only) muscle damage status. Interestingly, while cumulative exposure or large week-to-week changes in VHIR may represent a substantial increase in injury risk [5052], a high but gradual exposure to VHIR may confer additional protection to spikes in workload for soccer players [53].

While practitioners have reported the total distance covered as the most commonly tracked variable [8], our results show no evidence of any significant relationship with changes in muscle damage markers. In contrast to VHIR, low to moderate running intensities are thought to induce a lower magnitude of muscle damage [54], without significant perturbation in the membrane permeability [38, 55]. As a large proportion of the match-related total distance is covered at low intensity (e.g., walking, jogging) [56], this may explain an absence of a relationship with changes in muscle damage markers. Additionally, total distance has been largely correlated with salivary cortisol concentrations at G + $24 \mathrm{H}$ and $\mathrm{G}+48 \mathrm{H}$ in only one study (Tables 4 and 5) [57]. These relationships with residual endocrine responses would need to be confirmed by future investigations.

Our results displayed a large correlation (strong evidence) between VHIR and change in $\mathrm{CMJ}_{\mathrm{PPO}}$, yet at $\mathrm{G}+$ $24 \mathrm{H}$ only. This change in $\mathrm{CMJ}_{\mathrm{PPO}}$ was largely correlated with high accelerations at the same time-point but with moderate evidence due to the restricted number of studies considering this metric. These results highlight that the reduction in $\mathrm{CMJ}_{\mathrm{PPO}}$ at $\mathrm{G}+24 \mathrm{H}$, in addition to the increase in muscle damage markers, may be related to the repetitive stress (i.e., mechanical) sustained by the neuromuscular system throughout the frequent VHIR and high acceleration actions [58]. The amount of eccentric-related actions likely characterizes this mechanical stress potentially inducing changes in joint sequencing (e.g., increase in eccentric phase duration), in a motor pattern used for performance (adjustment of neuromuscular recruitment strategies) $[59,60]$ and selective damage of type II muscle fibers [61]. Accordingly, it has been recently determined that an increase by 0.6 $\mathrm{km}$ in VHIR, as a specific match-related intense activity, may induce a decrement in $\mathrm{CMJ}_{\mathrm{PPO}}$ by slightly more than the smallest worthwhile change (i.e., $1.0 \mathrm{~W} / \mathrm{kg}$ ) [62]. Consequently, the present meta-analytic impairment in $\mathrm{CMJ}_{\mathrm{PPO}}$ may reflect the power-based load characterized by VHIR during soccer matches. Conversely, total distance covered, including a low proportion of high-intensity eccentric actions, was not related to postmatch changes in $\mathrm{CMJ}_{\mathrm{PPO}}$.

Our results do not demonstrate evidence for significant relationships between any tracking variable and changes in post-match perceptual responses. While selfreport, perceptual measures such as questionnaires are simple and efficient methods [63] to assess matchrelated load, only a few studies have investigated such a relationship [21, 25, 32]. Today, a large majority of elite soccer clubs collect self-report measures (e.g., perceived recovery such as TQR) daily to monitor players' training-induced psychometric and wellbeing states [8]. However, they do not seem to use this monitoring tool as frequently for reflecting fatigue associated with performing home and away matches. This seems surprising since the match load represents the main determinant of a high weekly training load during the competitive season [64]. Particularly, subjective measures of mood disturbance, perceived stress and recovery may reflect acute 
and chronic loads with superior sensitivity and consistency in reference to more objective measures (e.g., muscle damage markers, $\mathrm{CMJ}_{\mathrm{PPO}}$ ) [65]. In our study, the lack of an association between subjective and objective measures provides support for the complementary inclusion of both measurements.

Practically, at $\mathrm{G}+24 \mathrm{H}$, for every $100 \mathrm{~m}$ of VHIR during a soccer match, CK activity would increase by $30 \%$ and $\mathrm{CMJ}_{\mathrm{PPO}}$ would decrease by $0.5 \%$ (Fig. 2). Our metaanalytic results show, for the first time, that VHIR appears as the strongest predictor of alterations in muscle damage and peak power output since it was the only tracking variable largely related to these biochemical and neuromuscular fatigue-related makers. The systematic and meta-analysis of the current literature suggests that the running distance covered above $5.5 \mathrm{~m} \mathrm{~s}^{-1}$, may explain up to $\sim 50 \%$ of the biochemical and neuromuscular post-match states. To date, other significant relationships between external load and post-match monitoring variables have yet to be determined for residual fatigue status especially at $\mathrm{G}+48 \mathrm{H}$ and $\mathrm{G}+72 \mathrm{H}$.

The strongest correlations between external load metrics (i.e., VHIR) and post-match muscle damage or $\mathrm{CMJ}_{\mathrm{PPO}}$ have been reported for acute fatigue (i.e., at Post) and at $\mathrm{G}+24 \mathrm{H}$ only, when magnitude of changes in most of the fatigue-related markers are at their greatest (i.e., peak changes) $[10,13,55]$. There was no evidence of any significant relationships at $\mathrm{G}+48 \mathrm{H}$ and $\mathrm{G}$ $+72 \mathrm{H}$. The restricted number of studies (10 studies) examining the multi-factorial nature of fatigue incurred post-soccer match limits the strength of some of our conclusions. Furthermore, given the complexity of fatigue causing mechanisms and those responsible for its reversal, biochemical and neuromuscular changes induced by a match exhibit considerable variability $[10,55$, 66]. Regarding muscle damage markers, and more specifically CK, part of the variability may be attributed to their rate of clearance from the circulation [67]. In addition, the players' aerobic fitness level [21] and specific neuromuscular characteristics (e.g., lower body strength) [68-71] have been associated with matchrelated activity and fatigue development. Additionally, players' degree of familiarization to eccentric training and actions (e.g., high decelerations) are believed to impact their recovery rate [72]. All these between-player discrepancies may explain weak correlations between some external load metrics (e.g., VHIR and/or acceleration patterns) and fatigue-related markers at $\mathrm{G}+48 \mathrm{H}$ and $\mathrm{G}+72 \mathrm{H}$.

One limitation of this present review with metaanalysis is the between-study differences in definitions regarding the main players' external load metrics. Indeed, while most of the selected studies tracked matchrelated sprinting efforts, only two of them applied a speed threshold above $25 \mathrm{~km} \mathrm{~h}^{-1}$, leading to a weaker strength of findings. As previously highlighted by others, gathering external load data from different tracking technologies and different products may induce some flaws (e.g., no agreed filtering methods, sampling rates, and data-processing algorithms across studies). As example, there can be substantial differences between products, particularly for threshold-based acceleration and deceleration variables [73, 74]. Finally, a relatively small number of studies was included in our analysis. Remarkably, all selected studies have been published over the last 7 years due to the recent technology development that allows collecting locomotor-related activities during competition. Additionally, these technologies are either mainly restricted to home matches (i.e., semiautomatic cameras or radio-frequency systems) or not allowed during official matches (i.e., GPS), while the situation is now evolving. Considering these limitations, further investigations would be needed to ascertain the strength of evidence regarding sprint $\left(>7 \mathrm{~m} \mathrm{~s}^{-1}\right)$, acceleration and deceleration variables. Moreover, further studies should investigate the use of individualized external load thresholds (based on players' physiological characteristics such as maximal aerobic speed and sprinting speed ) $[75,76]$ can more efficiently reflect the acute and residual changes in post-match muscle damage, neuromuscular and perceptual responses.

\section{Conclusions}

While total distance is likely the most commonly monitored variable in elite soccer, it is not associated with changes in any post-game fatigue-related markers. A unique finding of our meta-analysis, however, was the observation of large correlations between match-related VHIR (above $5.5 \mathrm{~m} \mathrm{~s}^{-1}$ ) distance and both acute (Post) and residual $(\mathrm{G}+24 \mathrm{H}$ but not $\mathrm{G}+48 \mathrm{H} / \mathrm{G}+72 \mathrm{H})$ changes in fatigue-related markers. Indeed, VHIR was identified as the only tracking variable that correlated largely with both biochemical and neuromuscular markers. Practically, at G $+24 \mathrm{H}$, for every $100 \mathrm{~m}$ of VHI running distance covered, $\mathrm{CK}$ activity would increase by $30 \%$ and $\mathrm{CMJ}_{\mathrm{PPO}}$ would decrease by $0.5 \%$. VHIR, at least when assessed during the first $24 \mathrm{~h}$ of the recovery process, represents the most sensitive tracking variable to depict biochemical and neuromuscular loads resulting from soccer match-play.

\section{Abbreviations}

Acc: Acceleration; BAM+: Brief assessment of the mood; CK: Creatine kinase; CMJ: Countermovement jump; $\mathrm{CMJ}_{\mathrm{pPO}}$ : Countermovement jump peak power output; conc: Concentric; Decel: Deceleration; DOMS: Delayed onset muscle soreness; ecc: Eccentric; GPS: Global positioning system; HIR: High-intensity running distance; KE: Knee extension; KF: Knee flexion; G + 24H: Measured $24 \mathrm{~h}$ the match; $\mathrm{G}+48 \mathrm{H}$ : Measured $48 \mathrm{~h}$ after the match; $\mathrm{G}+72 \mathrm{H}$ : Measured $72 \mathrm{~h}$ after the match; Mod: Moderate intensity running; MVIC: Maximal voluntary isometric contraction; Post: Measured after the match; PT: Peak 
torque; TD: Total distance covered; TQR: Total quality of recovery; VHIR: Very high-intensity running distance

\section{Acknowledgements}

Open Access funding provided by the Qatar National Library.

\section{Authors' contributions}

$\mathrm{KH}$ and JRS designed the study; $\mathrm{KH}$, JRS, MCR and MH gathered and organized the data; $\mathrm{KH}$ and JRS performed the quality assessment of the selected manuscripts and wrote the manuscript; $\mathrm{KH}$ analysed the data; $\mathrm{KH}$, MCR, MH, LPK, OG AND JRS assisted in the interpretation of data; $\mathrm{KH}, \mathrm{MCR}$, MH, LPK, OG AND JRS critically revised the manuscript; KH, MCR, MH, LPK, OG and JRS — read and approved the final manuscript.

\section{Funding}

No sources of funding were used to assist in the preparation of this article.

\section{Availability of Data and Materials}

The datasets used and/or analyzed during the current study are available from the corresponding author on reasonable request.

\section{Ethics Approval and Consent to Participate}

Not Applicable.

\section{Consent for Publication}

Not applicable.

\section{Competing Interests}

The authors, Karim Hader, Michael Rumpf, Maxime Hertzog, Liam Kilduff, Olivier Girard, and Joao Silva, declare that they have no competing interests.

\section{Author details}

${ }^{1}$ National Sports Medicine Programme, Excellence in Football Project, Aspetar - Qatar Orthopaedic and Sports Medicine Hospital, P.O BOX 29222 Doha, Qatar. ${ }^{2}$ Performance Department, Paris Saint-Germain F.C., Saint Germain-en-Laye, France. ${ }^{3}$ Auckland University of Technology, Sport Performance Research Institute New Zealand, Auckland, New Zealand. ${ }^{4}$ Footballscience.net, Roedermark, Germany. ${ }^{5}$ A-STEM, College of Engineering, Swansea University, Swansea, UK. ${ }^{6}$ Welsh Institute of Performance Science, Sport Wales, Cardiff, UK. ${ }^{7}$ Murdoch Applied Sport Science Laboratory, Murdoch University, Perth, Western Australia. ${ }^{8}$ Center of Research, Education, Innovation and Intervention in Sport (CIFI2D), Porto, Portugal.

\section{Received: 7 May 2019 Accepted: 2 October 2019}

\section{Published online: 09 December 2019}

\section{References}

1. Barnes C, Archer DT, Hogg B, Bush M, Bradley PS. The evolution of physical and technical performance parameters in the english premier league. Int J Sports Med. 2014;35(13):1095-100.

2. Buchheit M, Simpson BM. Player Tracking Technology: Half-Full or HalfEmpty Glass? Int J Sports Physiol Perform. 2016;14:1-23.

3. Scott D, Lovell R. Individualisation of speed thresholds does not enhance the dose-response determination in football training. J Sports Sci. 2018; 36(13):1523-32.

4. Buchheit M, Allen A, Poon TK, Modonutti M, Gregson W, Di Salvo V. Integrating different tracking systems in football: multiple camera semiautomatic system, local position measurement and GPS technologies. J Sports Sci. 2014 Dec;32(20):1844-57.

5. Wallace LK, Slattery KM, Coutts AJ. A comparison of methods for quantifying training load: relationships between modelled and actual training responses. Eur J Appl Physiol. 2014 Jan;114(1):11-20.

6. Buchheit M, Manouvrier C, Cassirame J, Morin JB. Monitoring Locomotor Load in Soccer: Is Metabolic Power, Powerful? Int J Sports Med. 2015; Dec; 36(14):1149-55.

7. Hader K, Mendez-Villanueva A, Palazzi D, Ahmaidi S, Buchheit M. Metabolic Power Requirement of Change of Direction Speed in Young Soccer Players: Not All Is What It Seems. PLoS One. 2016;11(3):e0149839.

8. Akenhead R, Nassis GP. Training Load and Player Monitoring in High-Level Football: Current Practice and Perceptions. Int J Sports Physiol and Perform. 2016;11(5):587-93.
9. Reilly T, Drust B, Clarke N. Muscle fatigue during football match-play. Sports Med. 2008;38(5):357-67.

10. Silva JR, Rumpf MC, Hertzog M, Castagna C, Farooq A, Girard O, et al. Acute and Residual Soccer Match-Related Fatigue: A Systematic Review and Metaanalysis. Sports Med. 2018; Mar;48(3):539-83.

11. Hader K, Palazzi D, Buchheit M. Change of direction speed in soccer: How much brake is enough? Kineziologija. 2015;47(1):67-74.

12. Russell M, Sparkes W, Northeast J, Cook CJ, Bracken RM, Kilduff LP. Relationships between match activities and peak power output and Creatine Kinase responses to professional reserve team soccer match-play. Hum Mov Sci. 2016 Feb;45:96-101.

13. Silva JR, Ascensao A, Marques F, Seabra A, Rebelo A, Magalhaes J. Neuromuscular function, hormonal and redox status and muscle damage of professional soccer players after a high-level competitive match. Eur J Appl Physiol. 2013;113(9):2193-201.

14. Silva JR, Rebelo A, Marques F, Pereira L, Seabra A, Ascensao A, et al. Biochemical impact of soccer: an analysis of hormonal, muscle damage, and redox markers during the season. Appl Physiol Nutr Metab. 2014;39(4):432-8.

15. Hader K, Mendez-Villanueva A, Williams B, Ahmaidi S, Buchheit M. Changes of direction during high-intensity intermittent runs: Neuromuscular and metabolic responses. BMC Sports Sci Med Rehabil. 2014;6(1):2.

16. Impellizzeri FM, Rampinini E, Marcora SM. Physiological assessment of aerobic training in soccer. J Sports Sci. 2005 Jun;23(6):583-92.

17. Young WB, Hepner J, Robbins DW. Movement demands in Australian rules football as indicators of muscle damage. J Strength Cond Res. 2012;26(2):492-6.

18. Magalhaes J, Rebelo A, Oliveira E, Silva JR, Marques F, Ascensao A. Impact of Loughborough Intermittent Shuttle Test versus soccer match on physiological, biochemical and neuromuscular parameters. Eur J Appl Physiol. 2010;108(1):39-48.

19. Colby MJ, Dawson B, Heasman J, Rogalski B, Gabbett TJ. Accelerometer and GPS-derived running loads and injury risk in elite Australian footballers. J Strength Cond Res. 2014;28(8):2244-52.

20. Duhig S, Shield AJ, Opar D, Gabbett TJ, Ferguson C, Williams M. Effect of high-speed running on hamstring strain injury risk. Br J Sports Med. 2016; 50(24):1536-40.

21. Draganidis D, Chatzinikolaou A, Avloniti A, Barbero-Alvarez JC, Mohr M, Malliou P, et al. Recovery kinetics of knee flexor and extensor strength after a football match. PloS One. 2015;10(6):e0128072.

22. Thorpe R, Sunderland C. Muscle damage, endocrine, and immune marker response to a soccer match. J Strength Cond Res. 2012;26(10):2783-90.

23. de Hoyo M, Cohen DD, Sanudo B, Carrasco L, Alvarez-Mesa A, Del Ojo JJ, et al. Influence of football match time-motion parameters on recovery time course of muscle damage and jump ability. J Sports Sci. 2016;34(14):1363-70.

24. Moher D, Liberati A, Tetzlaff J, Altman DG, Group P. Preferred reporting items for systematic reviews and meta-analyses: the PRISMA statement. Bmj. 2009;339:b2535.

25. Nedelec M, McCall A, Carling C, Legall F, Berthoin S, Dupont G. The influence of soccer playing actions on the recovery kinetics after a soccer match. J Strength Cond Res. 2014;28(6):1517-23.

26. Russell M, Northeast J, Atkinson G, Shearer DA, Sparkes W, Cook CJ, et al Between-Match Variability of Peak Power Output and Creatine Kinase Responses to Soccer Match-Play. J Strength Cond Res. 2015;29(8):2079-85.

27. Kmet LM, Lee RC, Cook LS. Standard quality assessment criteria for evaluating primary research papers from a variety of fields; 2004.

28. Aquino RL, Goncalves L, Vieira LH, Oliveira LP, Alves GF, Santiago PR, et al. Biochemical, physical and tactical analysis of a simulated game in young soccer players. J Sports Med Phys Fitness. 2016:56(12):1554-61.

29. Rampinini E, Bosio A, Ferraresi I, Petruolo A, Morelli A, Sassi A. Match-related fatigue in soccer players. Med Sci Sports Exerc. 2011;43(11):2161-70.

30. Romagnoli M, Sanchis-Gomar F, Alis R, Risso-Ballester J, Bosio A, Graziani RL, et al. Changes in muscle damage, inflammation, and fatigue-related parameters in young elite soccer players after a match. J Sports Med Phys Fitness. 2016; Oct:56(10):1198-205.

31. Scott A, Malone J, Morgans R, Burgess D, Gregson W, Morton J, et al. The relationship between physical match performance an 48-h post-game creatine kinase concentrations in English Premier League soccer players. Int J Sports Sci Coaching. 2016;11(6):846-52.

32. Shearer DA, Sparkes W, Northeast J, Cunningham DJ, Cook CJ, Kilduff LP. Measuring recovery: An adapted Brief Assessment of Mood (BAM+) compared to biochemical and power output alterations. J Sci Med Sport. 2017;20(5):512-7. 
33. Varley I, Lewin R, Needham R, Thorpe R, Burbeary R. Association between match activity variables, measures of fatigue and neuromuscular performance capacity following elite competitive soccer matches. J Human Kin. 2018:60:93-9.

34. Hopkins WG, Marshall SW, Batterham AM, Hanin J. Progressive statistics for studies in sports medicine and exercise science. Med Sci Sports Exerc. 2009 Jan; $41(1): 3-13$

35. van Tulder M, Furlan A, Bombardier C, Bouter L. Editorial Board of the Cochrane Collaboration Back Review G. Updated method guidelines for systematic reviews in the cochrane collaboration back review group. Spine. 2003;28(12):1290-9.

36. Furlan AD, Pennick V, Bombardier C, van Tulder M, Editorial Board CBRG. 2009 updated method guidelines for systematic reviews in the Cochrane Back Review Group. Spine. 2009;34(18):1929-41.

37. Higgins JP, Thompson SG, Deeks JJ, Altman DG. Measuring inconsistency in meta-analyses. Bmj. 2003;327(7414):557-60.

38. Brancaccio P, Lippi G, Maffulli N. Biochemical markers of muscular damage. Clin chem Lab Med : CCLM / FESCC. 2010;48(6):757-67.

39. Hedges L, Olkin I. Statistical methods for meta-analysis. 1985 ed. London: American Press; 1985.

40. Hunter JE, Schmidt FL. Methods ofmeta-analysis. In: Correcting Error and Bias in Research Findings. Newbury Park: Sage Publications; 1990.

41. Field AP. Meta-analysis of correlation coefficients: a Monte Carlo comparison of fixed- and random-effects methods. Psychol Methods. 2001 Jun;6(2):161-80.

42. Woods C, Hawkins R, Hulse M, Hodson A. The Football Association Medical Research Programme: an audit of injuries in professional football-analysis of preseason injuries. Br J Sports Med. 2002;36(6):436-41 discussion 41.

43. Opar DA, Williams MD, Timmins RG, Hickey J, Duhig SJ, Shield AJ. Eccentric hamstring strength and hamstring injury risk in Australian footballers. Med Sci Sports Exerc. 2015:47(4):857-65.

44. Woods C, Hawkins RD, Maltby S, Hulse M, Thomas A, Hodson A, et al. The Football Association Medical Research Programme: an audit of injuries in professional football--analysis of hamstring injuries. Br J Sports Med. 2004; 38(1):36-41.

45. Ekstrand J, Walden M, Hagglund M. Hamstring injuries have increased by 4\% annually in men's professional football, since 2001: a 13-year longitudinal analysis of the UEFA Elite Club injury study. Br J Sports Med. 2016 Jun;50(12):731-7.

46. Schache AG, Dorn TW, Blanch PD, Brown NA, Pandy MG. Mechanics of the human hamstring muscles during sprinting. Med Sci Sports Exerc. 2012 Apr; 44(4):647-58.

47. Chapman D, Newton M, Sacco P, Nosaka K. Greater muscle damage induced by fast versus slow velocity eccentric exercise. Int J Sports Med. 2006;27(8):591-8.

48. Friden J, Lieber RL. Eccentric exercise-induced injuries to contractile and cytoskeletal muscle fibre components. Acta Physiol Scand. 2001 Mar;171(3): 321-6.

49. Clarkson PM, Sayers SP. Etiology of exercise-induced muscle damage. Canadian J Appl Physiol. 1999;24(3):234-48.

50. Bowen L, Gross A, Gimpel M, Li F. Accumulated workloads and the acutechronic workload ratio relate to injury. Br J Sports Med. 2017;51(5):452-9.

51. Malone S, Owen A, Mendes B, Hughes B, Collins K, Gabbett TJ. High-speed running and sprinting as an injury risk factor in soccer: Can well-developed physical qualities reduce the risk? J Sci Med Sport. 2018;21(3):257-62.

52. Gabbett TJ. Debunking the myths about training load, injury and performance: empirical evidence, hot topics and recommendations for practitioners. Br J Sports Med. 2018;26.

53. Gabbett TJ, Nassis GP, Oetter E, Pretorius J, Johnston N, Medina D, et al. The athlete monitoring cycle: a practical guide to interpreting and applying training monitoring data. Br J Sports Med. 2017;51(20):1451-2.

54. Nosaka K, Newton M. Difference in the magnitude of muscle damage between maximal and submaximal eccentric loading. J Strength Con Res. 2002;16(2):202-8.

55. Brancaccio P, Maffulli N, Limongelli FM. Creatine kinase monitoring in sport medicine. Br Med Bull. 2007:81-82:209-30.

56. Stolen T, Chamari K, Castagna C, Wisloff U. Physiology of soccer: an update. Sports Med. 2005;35(6):501-36.

57. Romagnoli M, Sanchis-Gomar F, Alis R, Risso-Ballester J, Bosio A, Graziani RL, et al. Changes in muscle damage, inflammation, and fatigue-related parameters in young elite soccer players after a match. J Sports Med Phy Fitness. 2016;56(10):1198-205.
58. Silva JR, Magalhaes JF, Ascensao AA, Oliveira EM, Seabra AF, Rebelo AN. Individual match playing time during the season affects fitness-related parameters of male professional soccer players. J Strength Cond Res. 2011; 25(10):2729-39.

59. Kellis E, Kouvelioti V. Agonist versus antagonist muscle fatigue effects on thigh muscle activity and vertical ground reaction during drop landing. J Electromyogr Kinesio. 2009;19(1):55-64.

60. Rodacki AL, Fowler NE, Bennett SJ. Vertical jump coordination: fatigue effects. Med Sci Sports Exerc. 2002;34(1):105-16.

61. Clarkson PM, Tremblay I. Exercise-induced muscle damage, repair, and adaptation in humans. J Appl Physiol. 1988;65(1):1-6.

62. Morgans R, Di Michele R, Drust B. Soccer Match-Play Represents an Important Component of the Power Training Stimulus in Premier League Players. Int J Sports Physiol Perform. 2017;19:1-12.

63. Coutts AJ, Reaburn P. Monitoring changes in rugby league players' perceived stress and recovery during intensified training. Percept Mot Skills. 2008;106(3):904-16.

64. Los Arcos A, Mendez-Villanueva A, Yanci J, Martinez-Santos R. Respiratory and Muscular Perceived Exertion During Official Games in Professional Soccer Players. Int J Sports Physiol and Perform. 2016;11(3):301-4.

65. Saw AE, Main LC, Gastin PB. Monitoring the athlete training response: subjective self-reported measures trump commonly used objective measures: a systematic review. Br J Sports Med. 2016;50(5):281-91.

66. Lac G, Maso F. Biological markers for the follow-up of athletes throughout the training season. Pathologie-biologie. 2004;52(1):43-9.

67. Warren GL, O'Farrell L, Rogers KR, Billings KM, Sayers SP, Clarkson PM. CKMM autoantibodies: prevalence, immune complexes, and effect on CK clearance. Muscle Nerve. 2006;34(3):335-46.

68. Silva JR, Magalhaes J, Ascensao A, Seabra AF, Rebelo AN. Training status and match activity of professional soccer players throughout a season. $J$ Strength Cond Res. 2013;27(1):20-30.

69. Owen A, Dunlop G, Rouissi M, Chtara M, Paul D, Zouhal H, et al. The relationship between lower-limb strength and match-related muscle damage in elite level professional European soccer players. J Sports Sci. 2015:33(20):2100-5.

70. Silva JR, Nassis GP, Rebelo A. Strength training in soccer with a specific focus on highly trained players. Sports Med - Open. 2015 2015/04/02; 2(1):1-27.

71. Tofari P, Kemp J, Cormack S. A Self-Paced Team Sport Match Simulation Results In Reductions In Voluntary Activation And Modifications To Biological, Perceptual And Performance Measures At Half-Time, And For Up To 96 Hours Post-Match. J Strength Cond Res. 2018;32(12):3552-63.

72. Lavender AP, Nosaka K. A light load eccentric exercise confers protection against a subsequent bout of more demanding eccentric exercise. J Sci Med Sport. 2008;11(3):291-8.

73. Delaney JA, Cummins CJ, Thornton HR, Duthie GM. Importance, Reliability, and Usefulness of Acceleration Measures in Team Sports. J Strength Cond Res. 2018:32(12):3485-93.

74. Thornton HR, Nelson AR, Delaney JA, Serpiello FR, Duthie GM. Interunit Reliability abd Effect of Data-Processing Methods of Global Positioning Systems. Int J Sports Physiol Perform. 2019;14(4):432-8.

75. Abt G, Lovell R. The use of individualized speed and intensity thresholds for determining the distance run at high-intensity in professional soccer. J Sports Sci. 2009;27(9):893-8.

76. Lovell R, Abt G. Individualization of time-motion analysis: a casecohort example. Int J Sports Physiol Perform. 2013;8:456-8.

\section{Publisher's Note}

Springer Nature remains neutral with regard to jurisdictional claims in published maps and institutional affiliations. 